OPEN ACCESS

Edited by:

Marco Tafani,

Sapienza University of Rome, Italy

Reviewed by:

Zhenfeng Shi,

People's Hospital of Xinjiang Uygur Autonomous Region, China

André Zelanis,

Federal University of São Paulo, Brazil

${ }^{*}$ Correspondence:

Ying Xiang

xiang_ying22@163.com

Zhiwei Ma

704374245@qq.com

${ }^{\dagger}$ These authors have contributed equally to this work

Specialty section:

This article was submitted to Molecular and Cellular Oncology,

a section of the journal

Frontiers in Cell and Developmental

Biology

Received: 14 September 2021

Accepted: 03 November 2021

Published: 24 December 2021

Citation:

Lv D, Shen T, Yao J, Yang Q, Xiang Y and Ma Z (2021) HIF-1 $\alpha$ Induces

HECTD2 Up-Regulation and Aggravates the Malignant Progression

of Renal Cell Cancer via

Repressing miR-320a.

Front. Cell Dev. Biol. 9:775642.

doi: 10.3389/fcell.2021.775642

\section{HIF-1a Induces}

\section{HECTD2 Up-Regulation and}

Aggravates the Malignant Progression of Renal Cell Cancer via Repressing miR-320a

\author{
Dong Lv ${ }^{1 \dagger}$, Taimin Shen ${ }^{2 t}$, Juncheng Yao ${ }^{1}$, Qi Yang ${ }^{1}$, Ying Xiang ${ }^{1 *}$ and Zhiwei Ma ${ }^{3 *}$ \\ ${ }^{1}$ Department of Urology, Eastern Hospital, Sichuan Academy of Medical Sciences and Sichuan Provincial People's Hospital, \\ Chengdu, China, ${ }^{2}$ Health Management Center, Sichuan Academy of Medical Sciences and Sichuan Provincial People's Hospital, \\ Chengdu, China, ${ }^{3}$ Department of Urology, Sichuan Academy of Medical Sciences and Sichuan Provincial People's Hospital, \\ Chengdu, China
}

Renal cell carcinoma (RCC) is a frequent malignancy of the urinary system. It has been found that hypoxia mediates the malignant evolvement of RCC. Here, we probe the impact and potential mechanism of HECT domain E3 ubiquitin-protein ligase 2 (HECTD2) and HIF$1 a$ on regulating RCC evolvement. RCC tissues and adjacent normal tissues were collected, and the association between the expression profiles of HECTD2 and HIF-1a and the clinicopathological features was analyzed. Additionally, we constructed HECTD2/ HIF-1a overexpression and knockdown models in RCC cell lines to ascertain the impacts of HECTD2 and HIF-1a on RCC cell proliferation, apoptosis, migration, and growth in vivo. We applied bioinformatics to predict the upstream miRNA targets of HECTD2. Meanwhile, RNA immunoprecipitation (RIP), and the dual-luciferase reporter assays were employed to clarify the targeting association between HECTD2 and miR-320a. The effect of miR-320a on HECTD2-mediated RCC progression was investigated. The results suggested that both HIF-1 $\alpha$ and HECTD2 were up-regulated in RCC (compared with adjacent non-tumor tissues), and they had positive relationship. Moreover, higher level of HECTD2 and HIF-1a is associated with poorer overall survival of RCC patients. HECTD2 overexpression heightened RCC cell proliferation and migration, and weakened cell apoptosis. On the other hand, the malignant phenotypes of RCC cells were signally impeded by HECTD2 or HIF-1 a knockdown. Moreover, miR-320a targeted the $3^{\prime}$-untranslated region of HECTD2 and suppressed HECTD2 expression. The rescue experiments showed that miR-320a restrained HECTD2-mediated malignant progression in RCC, while up-regulation of HIF1a hampered miR-320a expression. Collectively, HIF-1 a mediated HECTD2 up-regulation and aggravated RCC progression by attenuating miR-320a.

Keywords: HIF-1 $\alpha$, HECTD2, MiR-320a, renal cell carcinoma, progression 


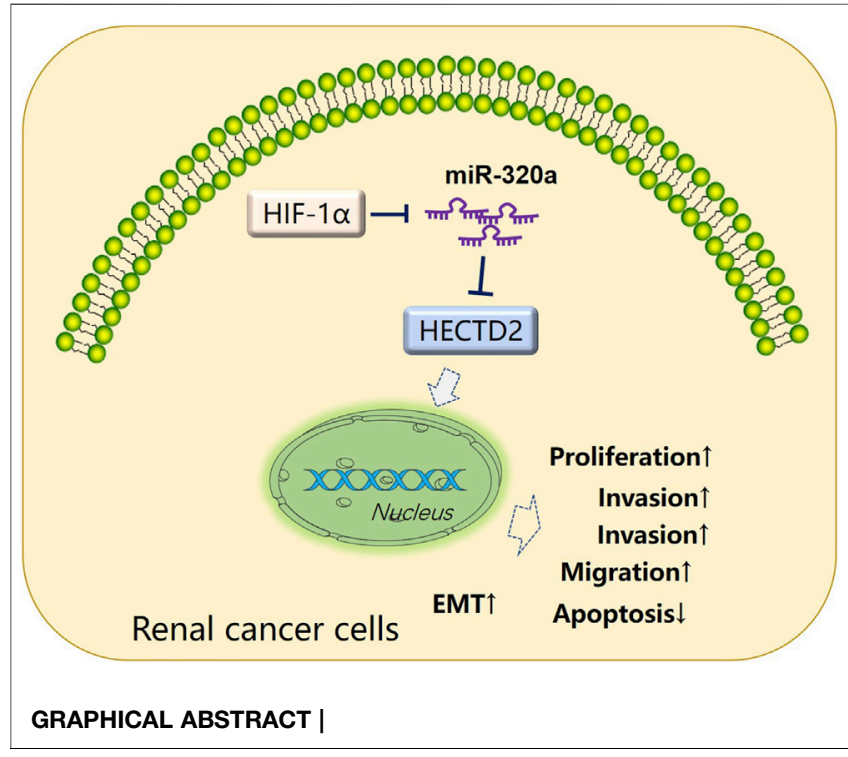

Graphical abstract: HIF-1 $\alpha$ enhances HECTD2 expression by attenuating miR-320a, thereby boosting RCC cell proliferation, migration, invasion, and EMT and abating apoptosis, which accelerates the malignant progression of RCC.

\section{INTRODUCTION}

Renal cell cancer (RCC) is the third most familiar genitourinary malignancy globally, with a high mortality rate (Shingarev and Jaimes, 2017). Clear cell renal cell carcinoma (ccRCC) is a familiar subtype of RCC, accounting for approximately $75 \%$ of all RCC cases (Song et al., 2018; Wolf et al., 2020). Over the last few years, treatment of RCC has improved with advances in medical technology, but the prognosis of RCC patients remains poor due to its high recurrence and metastasis rate (Wu et al., 2014; Di Franco et al., 2020). Therefore, it's indispensable to further probe the molecular regulatory mechanism of the occurrence and development of RCC and provide new biomarkers for the targeted therapy.

microRNAs (miRNAs) are processed from pri-miRNAs. They are highly conserved endogenous single-stranded non-coding small RNAs with small fragments of 20-24 nucleotides in length (Saliminejad et al., 2019). Studies have manifested that miRNA functions as a post-transcriptional regulator that can modulate cancer evolvement by combining with specific sequences of $3^{\prime}$ UTR of the target genes and impeding the expression of these genes (Ferragut Cardoso et al., 2020). It is reported that miRNAs are extensively involved in RCC development. For example, it has been revealed that miR-340 is up-regulated in RCC, which accelerates RCC cell proliferation, migration and invasion by hampering the FZD3 profile (Xiang et al., 2021). Additionally, miR-31-5p is lowly expressed in RCC, and it attenuates the malignant behaviors of RCC cells by targeting and reversely modulating HMGA1 (Liu et al., 2021). Also, Cheng $\mathrm{C}$ et al. have found that miR-26a-5p is knocked down in RCC, and it curbs RCC cell proliferation and invasion and intensifies cell death by targeting E2F7 (Cheng et al., 2020). miR320a has been found sponged by lncRNA CCAT2 (Wu et al., 2020), but its downstream mechanism in RCC progression needs further investigation.

Hypoxia is among the usual pathological conditions in which oxygen is restricted in tissues, and it facilitates tumor cell invasion and migration, impedes apoptosis, and is associated with tumor metastasis (Rankin et al., 2016). RCC, originating from renal proximal tubular epithelial cells, is one of the most frequent cancers that are highly sensitive to hypoxia (Schödel et al., 2016). Hypoxia-inducible factor $1 \alpha$ (HIF-1a), a functional subunit of HIF-1, has been confirmed to be extensively involved in RCC evolvement as an essential transcription factor that exerts an active role in hypoxia (Gudas et al., 2014). Besides, it has been revealed that apoptosis inhibition induces cancer cell proliferation and metastasis under hypoxia (Ge et al., 2018). Homologous to E6AP C-terminus (HECT) E3 ubiquitin ligase (HECTD) members have been found involved in tumor progression through ubiquitin modification (Jiang et al., 2020). For example, HECTD1 downregulation enhances cervical cell migration and epithelialmesenchymal transition by stabilizing SNAIL in a ubiquitination regulation manner (Wang et al., 2020). HETCD2 is a kind of E3 ubiquitin ligase located at 10q23.32, with 4,874 bp in length. Similar to the other E3 ubiquitin ligases, HECTD2 ubiquitinated and induced protein inhibitor of activated STAT 1 (PIAS1), thus contributing to innate immunity and experimental lung injury followed by pneumonia (Coon et al., 2015). More importantly, HECTD2 also participates in neuroblastoma evolvement (Suo et al., 2018). Nevertheless, further research is needed to determine whether HECTD2 has any significant value in RCC.

By detecting the expression of HIF-1 $\alpha$ and HECTD2 in RCC tissues and cells, we discovered that HIF-1 $\alpha$ and HECTD2 were overexpressed in RCC and had a positive relationship. HIF-1a boosted HECTD2 expression and facilitated the malignant biological behaviors of RCC. In addition, we adopted bioinformatics analysis and the result revealed a targeted relationship between miR-320a and HECTD2, whereas HIF-1a inhibited miR-320a. Thus, we supposed that there's a novel HIF$1 \alpha / \mathrm{miR}-320 \mathrm{a} / \mathrm{HECTD} 2$ axis in RCC progression. In conclusion, this study uncovers a new molecular mechanism in RCC and provides a new theoretical reference for RCC therapy.

\section{MATERIALS AND METHODS}

\section{Patient Tissue Samples}

This experiment was authorized by the Ethics Committee of Sichuan Provincial People's Hospital and implemented in accordance with the Helsinki Declaration. RCC tissues were obtained from 104 RCC patients $(45.0 \pm 12.3$ years old $)$ who underwent surgical excision but did not receive chemotherapy or radiotherapy. The matched paracancerous normal tissues were also obtained from the same patients, which were 3-4 cm away from the tumorous tissues, and no cancer cells were discovered in the postoperative pathological examination. All tissues were frozen in liquid nitrogen and stored at $80^{\circ} \mathrm{C}$ for subsequent 
TABLE 1 | Association between the expression of HECTD2 and clinical features in tissue samples of patients with RCC.

\begin{tabular}{|c|c|c|c|c|}
\hline \multirow[t]{2}{*}{ Characteristics } & \multirow[t]{2}{*}{ Patients } & \multicolumn{2}{|c|}{ Expression of HECTD2 } & \multirow[t]{2}{*}{$p$-value } \\
\hline & & Low-HECTD2 & High-HECTD2 & \\
\hline Total & 104 & 49 & 55 & - \\
\hline Age (years) & - & - & - & 0.599 \\
\hline$<60$ & 58 & 26 & 32 & - \\
\hline$\geq 60$ & 46 & 23 & 23 & - \\
\hline Gender & - & - & - & 0.207 \\
\hline Male & 66 & 28 & 38 & - \\
\hline Female & 38 & 21 & 17 & - \\
\hline Tumor stage & - & - & - & $0.021^{*}$ \\
\hline pT1 & 47 & 28 & 19 & - \\
\hline pT2/T3 & 57 & 21 & 36 & - \\
\hline Fuhrman grade & - & - & - & $0.034^{*}$ \\
\hline $1+\|$ & 48 & 28 & 20 & - \\
\hline $\mathrm{III}+\mathrm{IV}$ & 56 & 21 & 35 & - \\
\hline Tumor diameter (cm) & - & - & - & 0.451 \\
\hline$\leq 7 \mathrm{~cm}$ & 55 & 24 & 31 & - \\
\hline$>7 \mathrm{~cm}$ & 49 & 25 & 24 & - \\
\hline Lymph node metastasis & - & - & - & 0.209 \\
\hline Negative & 68 & 29 & 39 & - \\
\hline Positive & 36 & 20 & 16 & - \\
\hline
\end{tabular}

TABLE 2 | Correlation between HIF-1a expression and clinical features in RCC patients.

\begin{tabular}{|c|c|c|c|c|}
\hline \multirow[t]{2}{*}{ Characteristics } & \multirow[t]{2}{*}{ Patients } & \multicolumn{2}{|c|}{ Expression of HIF-1a } & \multirow[t]{2}{*}{$p$-value } \\
\hline & & Low- HIF-1a & High-HIF-1a & \\
\hline Total & 104 & 53 & 51 & - \\
\hline Age (years) & - & - & - & 0.876 \\
\hline$<60$ & 64 & 33 & 31 & - \\
\hline$\geq 60$ & 40 & 20 & 20 & - \\
\hline Gender & - & - & - & 0.698 \\
\hline Male & 53 & 28 & 25 & - \\
\hline Female & 51 & 25 & 26 & - \\
\hline Tumor stage & - & - & - & $0.048^{\star}$ \\
\hline pT1 & 23 & 30 & 19 & - \\
\hline рT2/T3 & 19 & 23 & 32 & - \\
\hline Fuhrman grade & - & - & - & $0.049^{\star}$ \\
\hline $1+\|$ & 51 & 31 & 20 & - \\
\hline $\mathrm{III}+\mathrm{IV}$ & 53 & 22 & 31 & - \\
\hline Tumor diameter (cm) & - & - & - & 0.708 \\
\hline$\leq 7 \mathrm{~cm}$ & 57 & 30 & 27 & - \\
\hline$>7 \mathrm{~cm}$ & 47 & 23 & 24 & - \\
\hline Lymph node metastasis & - & - & - & 0.838 \\
\hline Negative & 50 & 26 & 24 & - \\
\hline Positive & 54 & 27 & 27 & - \\
\hline
\end{tabular}

(Note: ${ }^{*} \mathrm{p}<0.05$, the difference was statistically significant).

testing. All patients participating in this study signed informed consent. The general information of patients is shown in Tables 1, 2.

\section{Cell Culture and Transfection}

RCC cell lines 786-O and A-498 were bought from the America Type Culture Collection (ATCC, Manassas, VA, United States). Cells were grown in the DMEM (Thermo Fisher Scientific, Waltham, MA, United States) comprising 10\% fetal bovine serum, $100 \mathrm{U} / \mathrm{mL}$ penicillin (Invitrogen, Carlsbad, CA, United
States) and $100 \mu \mathrm{g} / \mathrm{ml}$ streptomycin (Invitrogen) and maintained at $37^{\circ} \mathrm{C}$ in a $5 \% \mathrm{CO}_{2}$ incubator. Cells in the logarithmic growth stage were trypsinized before being seeded into 6 -well plates $(5 \times$ $10^{6}$ cells/well). The transfection was made after cell growth was stable. 786-O and A-498 cells were transfected with HECTD2 overexpression and knockdown plasmids, HIF-1a overexpression plasmids, miR-320a mimics/inhibitors and the corresponding negative controls. Afterward, they were incubated at $37^{\circ} \mathrm{C}$ with $5 \% \mathrm{CO}_{2}$. After transfection for $24 \mathrm{~h}$, the cells were employed for subsequent experiments. The knockdown sequences of HECTD2: si-HECTD2, AUAAAACUGCUGAAAGUGGAA; si-NC: TTC TCCGAACGTGTCACGT.

\section{Colony Formation Assay}

Cells were inoculated into 6-well plates (500 cells/well), and the culture solution was altered every 5 days. After incubation for 7-14 days, cells were rinsed with PBS, immobilized with $4 \%$ paraformaldehyde, and dyed with $0.5 \%$ crystal violet. At last, the colony formation number was counted using Olympus microscope (BX23, Japan).

\section{5-Bromodeoxyuridine (BrdU) Staining}

The BrdU cell proliferation detection kit (Wuhan AmyJet Scientific Inc. Wuhan, China) was applied for cell proliferation assessment. 786-O and A-498 cells were seeded into 96-well plates $\left(6 \times 10^{4}\right.$ cells $\left./ \mathrm{mL}\right)$, respectively and incubated overnight with the constant temperature at $37^{\circ} \mathrm{C}$ and $5 \% \mathrm{CO}_{2}$ environment. The next day, each well was supplemented with $20 \mu \mathrm{L} \mathrm{BrdU}$ $(50 \mathrm{mM})$ and incubated for another $2 \mathrm{~h}$. The cells were fixed by 4\% Paraformaldehyde for $30 \mathrm{~min}$ at room temperature, and incubated with peroxidase-coupled anti-BrdU-antibody (Sigma-Aldrich) for $90 \mathrm{~min}$ at $37^{\circ} \mathrm{C}$. After being cleaned with PBS, the cells were incubated with DAPI (Beyotime, Shanghai, China) for 5 min at temperature. Subsequently, the BrdU-positive 


\section{TABLE 3 |}

Primer sequence of

gene name $\left(5^{\prime} \rightarrow 3^{\prime}\right)$

HECTD2

HIF-1 $1 \alpha$

miR-320a

U6

GAPDH

cells were counted under a fluorescence microscope (Olympus, Japan).

\section{Transwell Experiment}

Cell migration and invasion were determined by the Transwell assay. Cells were put in the Transwell upper chamber at $2 \times 10^{4}$ cells/well, and the lower chamber was supplemented with $600 \mu \mathrm{L}$ culture medium containing $20 \%$ FBS and cultured at $37^{\circ} \mathrm{C}$. Twelve hours later, the upper chamber cells were removed, immobilized with $4 \%$ paraformaldehyde, dyed with $0.1 \%$ crystal violet, dried, photographed and quantified. The invasion assay was the same as the migration assay, except that the upper chamber of the Transwell was pre-coated with Matrigel before adding the cells.

\section{Flow Cytometry}

The Annexin V-FITC/PI Apoptosis Detection Kit (Yeason, Beijing, China) was used for evaluating the apoptosis of RCC cells. Briefly, the RCC cells were detached with $0.25 \%$ trypsin without EDTA and collected via centrifugation $\left(300 \mathrm{~g}\right.$, at $4^{\circ} \mathrm{C}$ for $\left.5 \mathrm{~min}\right)$. Next, the cells were washed twice with pre cooled PBS and centrifuged at $4^{\circ} \mathrm{C}$ for $5 \mathrm{~min}(300 \mathrm{~g})$. $100 \mu \mathrm{L} 1 \times$ Binding buffer was used to suspend the cells, followed by incubating with $5 \mu \mathrm{L}$ Annexin V-FITC and $10 \mu \mathrm{L}$ PI stabilizing solution. After a 10 -min incubation at room temperature, $400 \mu \mathrm{L} 1 \times$ Binding Buffer was added and the apoptosis was detected by flow cytometry at $488 \mathrm{~nm}$ (BD Biosciences, Franklin Lakes, NJ, United States).

\section{Real-Time Quantitative PCR}

Total RNA was separated from 786-O and A-498 cells by applying the Trizol reagent. RNA was reversely transcribed into cDNA with the PrimeScript ${ }^{\mathrm{TM}}$ RT Reagent kit (Invitrogen, Shanghai, China) per the manufacturer's guidelines. qPCR was implemented using the Bio-Rad CFX96 quantitative PCR system and SYBR, with predenaturation at $95^{\circ} \mathrm{C}$ for $5 \mathrm{~min}$, followed by denaturation at $95^{\circ} \mathrm{C}$ for $15 \mathrm{~s}$ and annealing at $60^{\circ} \mathrm{C}$ for $30 \mathrm{~s}$. U6 served as an endogenous control of miR-320a, and GAPDH was that of HECTD2 and HIF-1a. The $2^{(-\Delta \Delta C t)}$ method was adopted for statistics. Each test was done three times. Primer sequences are exhibited in Table 3.

\section{Western Blot}

The cells were treated, and the culture medium was removed. Then, the protein lysate (Roche) was added for total protein separation. $50 \mu \mathrm{g}$ total protein was subjected to $12 \%$ polyacrylamide gel and went through $2 \mathrm{~h}$ of electrophoresis at $100 \mathrm{~V}$. It was then transferred to polyvinylidene fluoride (PVDF) membranes. After being blocked with 5\% skimmed milk at RT for $1 \mathrm{~h}$, the membranes were rinsed with TBST three times $(10 \mathrm{~min}$ each) and incubated with the primary antibodies (1:1,000) of anti-Bax (ab182734), anti-P53 (ab32389), anti-Caspase3 (ab32351), anti-P21 (ab109199), anti-HIF-1a (ab179483), anti-HECTD2 (ab249770), anti-Ecadherin (ab40772), anti-Vimentin (ab92547), and anti-Ncadherin (ab76011) overnight at $4^{\circ} \mathrm{C}$. After washing the membranes with TBST, we incubated them with horseradish peroxidase (HRP)-labeled Goat anti-Rabbit IgG (ab205718) $(1: 2,500)$ for $1 \mathrm{~h}$ at RT. The above antibodies were obtained from Abcam (Cambridge, United Kingdom). Afterward, the membranes were cleaned with TBST 3 times $(10 \mathrm{~min}$ each). Finally, the ECL chromogenic agent (Millipore, Bedford, MA, United States) was employed for exposure and imaging with a membrane scanner.

\section{Dual-Luciferase Reporter Assay}

PmirGLO vectors (Promega, United States) containing miR-320a sequences with wild-type or mutant binding locations for HECTD2 (HECTD2-WT, HECTD2-MT) were adopted. 786-O and A-498 cells were co-transfected with miR-320a mimics and the luciferase vectors. Detection of luciferase activity was made with a Dual-luciferase Reporter Gene Assay System (Promega, United States).

\section{RNA Immunoprecipitation}

RIP was implemented with Anti-AGO2 (\#03-110, Millipore, Germany) by applying the Magna RIP RNA binding protein immunoprecipitation kit (Millipore, Germany). The RNA binding complex was analyzed by qRT-PCR. Anti-IgG served as an isotype control.

\section{Tumor Formation in Nude Mice}

BALB/c-nu nude mice (six-week-old) were chosen to construct an in vivo tumorigenic model. Logarithmic growth phase 786-O and A- 498 cells were obtained by $0.25 \%$ trypsin and resuspended in the serum-free medium to fabricate single-cell suspensions $(3 \times$ $10^{7}$ cells $/ \mathrm{mL}$ ). Next, $0.1 \mathrm{ml}$ cell suspension was injected subcutaneously into the left forelimb armpit of each nude mouse (70 mice in total, 10 mice in each group). After injection, tumors were taken from mice under anesthesia on day $7,14,21,28$, and 35 , respectively and weighed. The longest diameter of the tumor 1) and the shortest diameter perpendicular to it 2) were monitored with vernier calipers, and the tumor volume $\mathrm{V}\left(\mathrm{mm}^{3}\right)=0.5 \times \mathrm{a} \times \mathrm{b}^{2}$. All experiments were authorized by the Animal Experimentation Ethics Review Committee of the Sichuan Provincial People's Hospital. Experiments were implemented strictly following the National Institutes of 

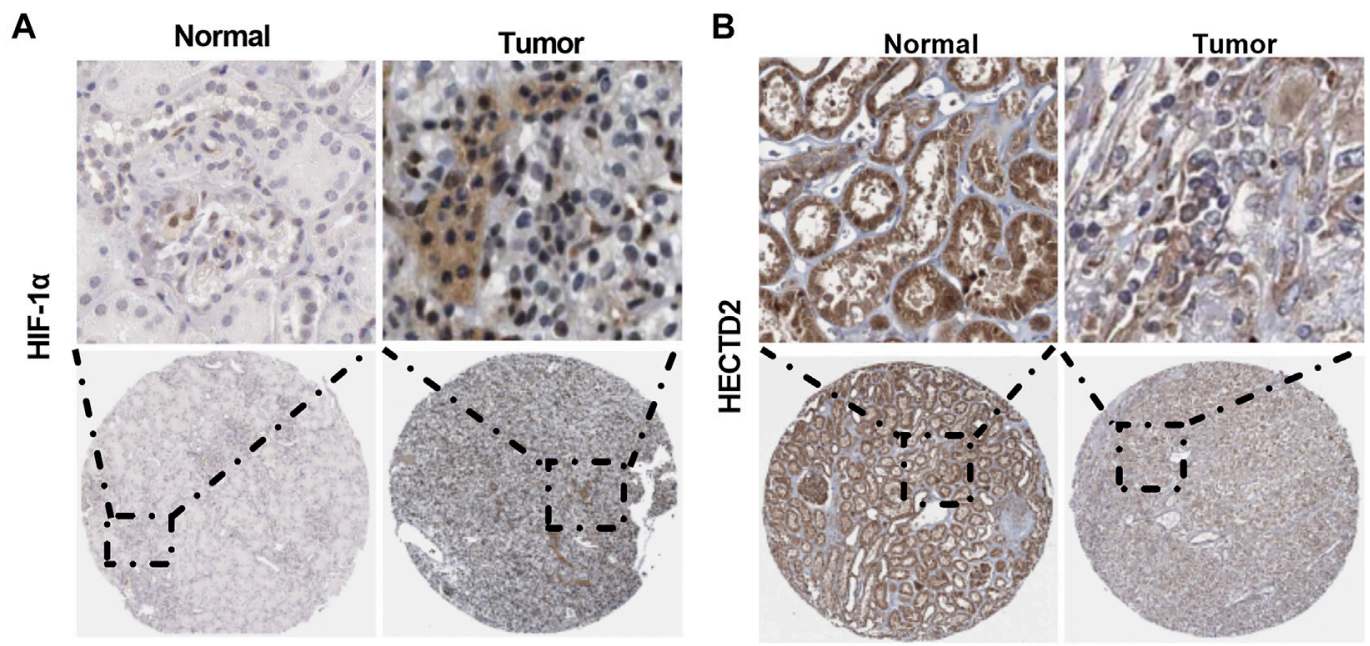

C
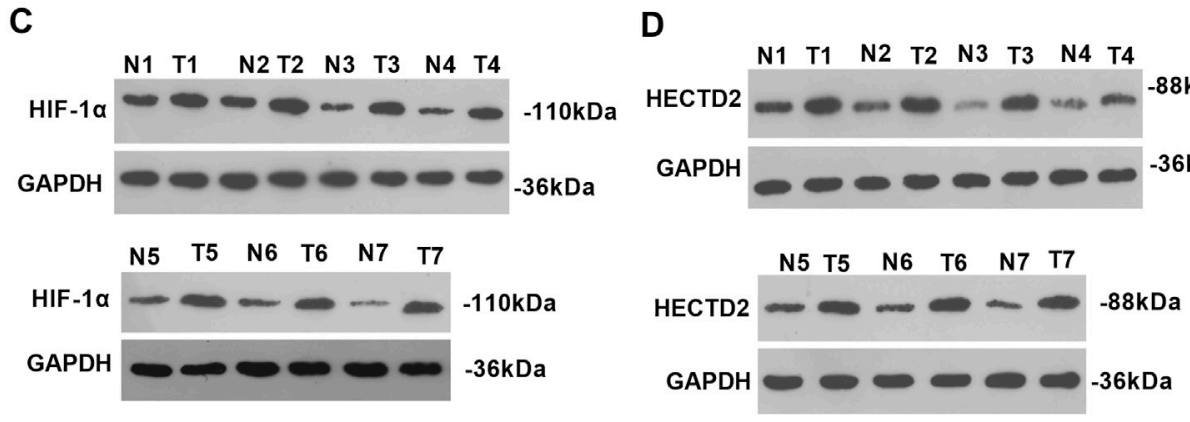

E

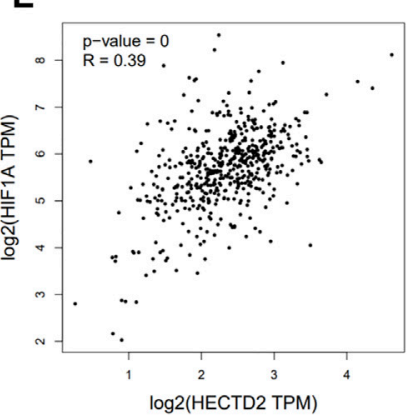

$\mathbf{F}$
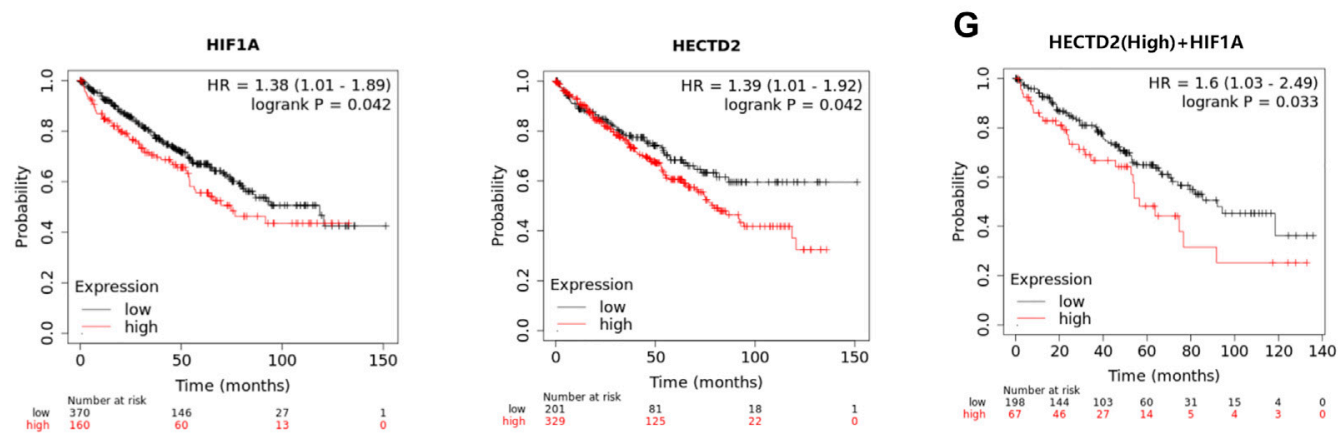

FIGURE 1 | Expression and prognosis of HIF-1 $\alpha$ and HECTD2 in RCC (A,B). The number of HIF-1 $\alpha$ and HECTD2 positive cells in RCC tissue and normal paracancerous tissues was analyzed in the Human Protein Atlas database (https://www.proteinatlas.org/). (C,D): HIF-1a and HECTD2 protein profiles in 7 cases of RCC tissues (T) and normal paracancerous tissues (N) were compared by WB. (E) The online database GEPIA was used for analyzing the expression relationship in KIRC (http://gepia.cancer-pku.cn/). (F,G): Prognostic analysis of HIF-1a and HECTD2 in RCC was performed on Kaplan-Meier Plotter (http://kmplot.com/analysis/).

Health Guidelines for the Care and Use of Laboratory Animals (NIH Publication No. 8023).

\section{TdT-Mediated dUTP Nick End Labeling Staining}

As described previously, paraffin sections of tumor tissues were made, dewaxed with toluene, and then dehydrated with different gradients of ethanol following the standard procedure. TUNEL staining of tumor tissue was then performed using a fluorescent assay kit (Cat\#
11684817910, Roche, Shanghai, China) according to the manufacturer's guidelines.

\section{Immunohistochemistry}

RCC tissue specimens were sectioned by conventional paraffin embedding $(4 \mu \mathrm{M})$, dewaxed with conventional xylene, hydrated with gradient ethanol, and inactivated with $3 \% \mathrm{H}_{2} \mathrm{O}_{2}$ for $10 \mathrm{~min}$. Microwave repair was made by applying $0.01 \mathrm{~mol} / \mathrm{L}$ sodium citrate buffer $(\mathrm{pH}=6.0,15 \mathrm{~min})$. Afterward, the sections were closed with $5 \%$ bovine serum albumin (BSA) for $20 \mathrm{~min}$, followed by incubation with the Ki67 antibody (ab15580, Abcam, MA, 
United States) overnight at $4^{\circ} \mathrm{C}$. On day 2, the Goat-anti-Rabbit IgG was added and incubated at RT for $20 \mathrm{~min}$, cleaned with PBS and imaged with DAB. The sections were inhibited by hematoxylin, dehydrated, and transparentized, and the closure was observed under the microscope.

\section{Tissue Immunofluorescence}

Paraffin tissue sections were dewaxed and hydrated. After antigen repair, the sections were sealed and maintained with the primary antibodies, including anti-HIF-1 $\alpha$ (ab179483, Abcam) and antiHECTD2 (HPA037767, Sigma-Aldrich). After being washed with PBS, the Goat polyclonal Secondary Antibody to Rabbit IgGH\&L (Alexa Fluor ${ }^{\circledR} 488$ ) (ab150077) was added for incubation. Afterward, 4,6 diamidinyl-2-phenyl indole (DAPI) nuclear staining was conducted, and the sections were blocked, observed, and photographed. Three sections were chosen from each specimen, and five non-overlap high-power fields of cancer tissues were randomly chosen from each section. The number of HIF-1 $\alpha$ and HECTD2 staining positive cells in each field of view was counted using Image-Pro Plus image analysis software, and the number of HIF-1 $\alpha$ and HECTD2 staining positive cells $/ \mathrm{mm}^{2}$ was calculated.

\section{Statistical Analysis}

SPSS22.0 statistical software (SPSS Inc., Chicago, IL, United States) was employed for statistical analysis. All data were presented as mean \pm SD. Measurement data were expressed as mean \pm standard deviation ( $\mathrm{x} \pm \mathrm{s}$ ), and enumeration data were expressed as four tables (or percentages). $\chi^{2}$ was adopted to compare differences between the two groups. One-way ANOVA was employed for the multifactor comparison, and the comparison between two groups was made by $t$ test. $p<0.05$ represented statistical significance.

\section{RESULTS}

\section{Expression and Prognosis of HIF-1a and HECTD2 in RCC}

To investigate whether HIF-1a and HECTD2 contribute to RCC progression, we consulted the database (https://www. proteinatlas.org/) and discovered that HIF-1 $\alpha$ and HECTD2 levels were both highly expressed in RCC tissues (Figures 1A,B). Also, WB results uncovered that HIF-1a and HECTD2 expression levels were significantly higher in tumor tissues of RCC patients than those in corresponding surrounding non-tumor tissues (Figures 1C,D). More importantly, Fuhrman grade in patients with higher profiles of HIF- $1 \alpha$ and HECTD2 was generally staged III + IV and tumor stage tended to be pT2/T3 (Tables 1, 2). Besides, there is a positive relationship between HIF-1a expression and HECTD2 expression in KIRC (analyzed by GEPIA) (Figure 1E). Meanwhile, patients with high expression of HIF-1 $\alpha$ and HECTD2 had worse prognosis compared with those with low expression of HIF-1a and HECTD2. Additionally, those patients with higher HECTD2+higher HIF-1a had poorer overall survival of those with higher
HECTD2+lower HIF-1a $(p=0.032)$ [data analyzed via Kaplan-Meier Plotter (http://kmplot.com/analysis/)] (Figures $\mathbf{1 F}, \mathbf{G})$. These results prompted that HIF-1 $\alpha$ and HECTD2 were associated with the malignant phenotype of RCC cells and might exert carcinogenic effects.

\section{Impacts of Overexpressing HECTD2 on the Malignant Phenotypes of RCC in vitro}

We constructed the HECTD2 overexpression model in 786-O and A-498 cell lines and tested the transfection effect by RTqPCR to probe the influence of HECTD2 on the malignant progression of RCC ( $p<0.05$, Figure 2A). Meanwhile, the detection of RCC cell proliferation and apoptosis was made using the colony formation experiment, BrdU assay and flow cytometry, respectively. Interestingly, overexpressing HECTD2 significantly motivated proliferation and bridled apoptosis in RCC $(p<0.05$, Figures 2B-D). Additionally, $\mathrm{WB}$ results illustrated that the pro-apoptotic proteins $\mathrm{P} 53$, Bax, p21, and c-Caspase3 were significantly down-regulated after HECTD2 overexpression ( $p<0.05$, Figure 2E). Besides, Transwell assay results testified that overexpressing HECTD2 significantly facilitated RCC cell migration and invasion $(p<$ 0.05, Figure 2F). Also, WB results signified that overexpressing HECTD2 significantly repressed the expression of the epithelial marker E-cadherin and elevated the levels of interstitial markers Vimentin and $\mathrm{N}$-cadherin $(p<0.05$, Figure 2G). These findings confirmed that overexpressing HECTD2 intensified the malignant progression of RCC.

\section{Influence of HECTD2 Overexpression on the Malignant Phenotype of RCC in vivo}

We constructed a HECTD2 overexpression model in 786-O and A-498 cells to testify the influence of HECTD2 on tumor growth in vivo and found that overexpressing HECTD2 significantly heightened tumor volume and weight $(p<$ 0.05 , Figures $3 \mathrm{~A}-\mathrm{C})$. Also, IHC revealed a substantial elevation in the Ki67-positive cell number in 786-O and A-498 cells after HECTD2 overexpression $(p<0.05$, Figure 3D). Moreover, WB results demonstrated that overexpressing HECTD2 in 786-O and A-498 cells significantly facilitated the HECTD2 profile in the transplanted tumor tissues $(p<0.05$, Figure 3E). The tissue immunofluorescence data exhibited that the fluorescence intensity of the HECTD2 group was more obvious than that of the control group $(p<0.05$, Figure 3F). These results added to evidence that overexpressing HECTD2 boosted RCC cell growth.

\section{HIF-1a Expedited the Expression of HECTD2 and Its Malignant Phenotype}

To ascertain the function of HIF-1 $\alpha$ and HECTD2 in RCC evolvement, we knocked down HECTD2 on the basis of HIF1a overexpression in 786-O cells and monitored the 
A

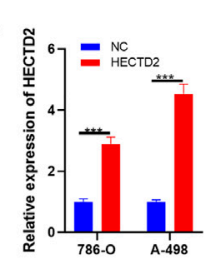

B
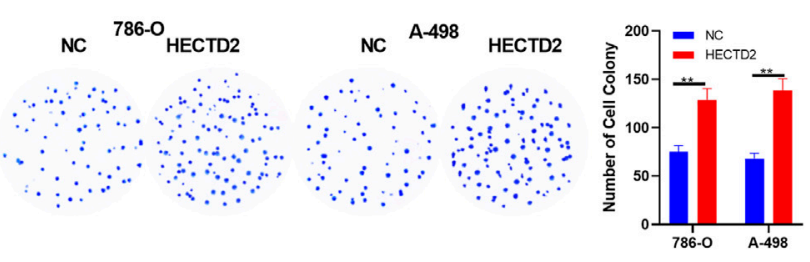

C
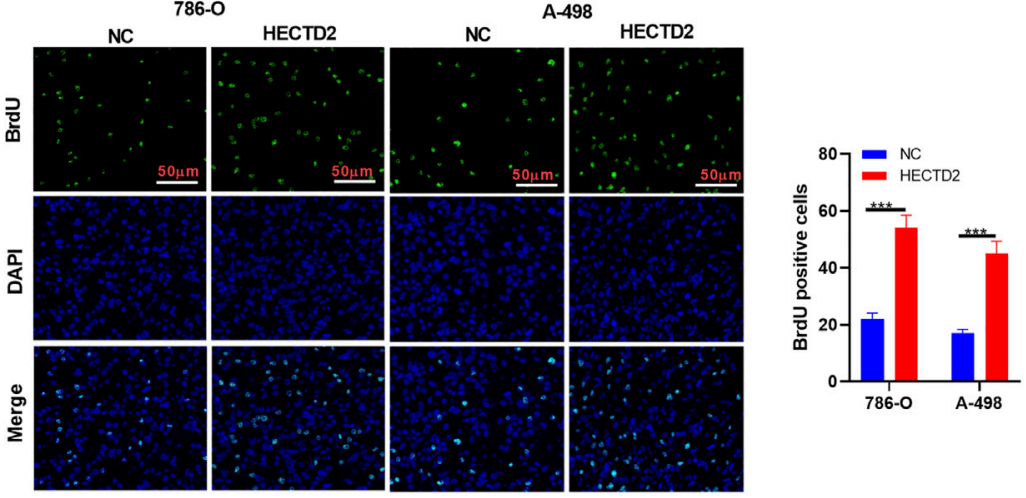

D
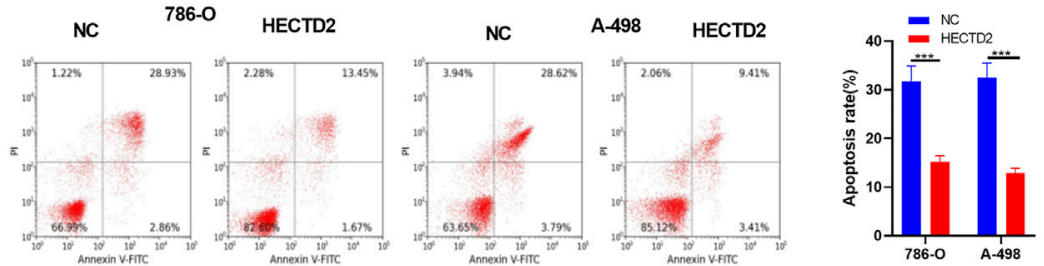

E
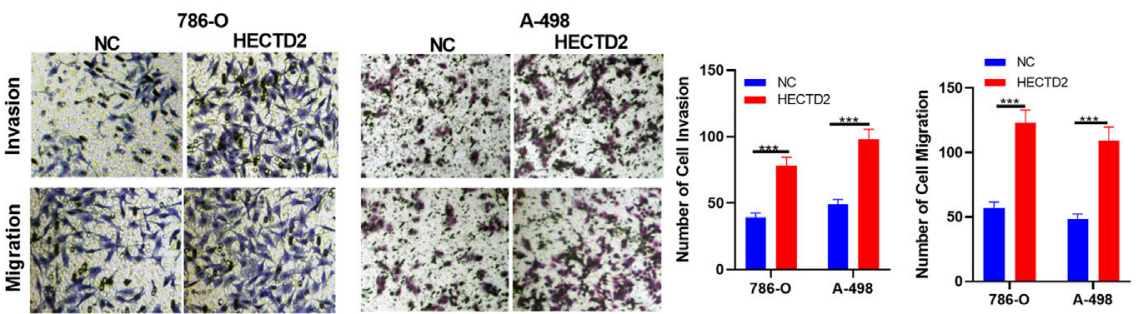

$\mathbf{F}$
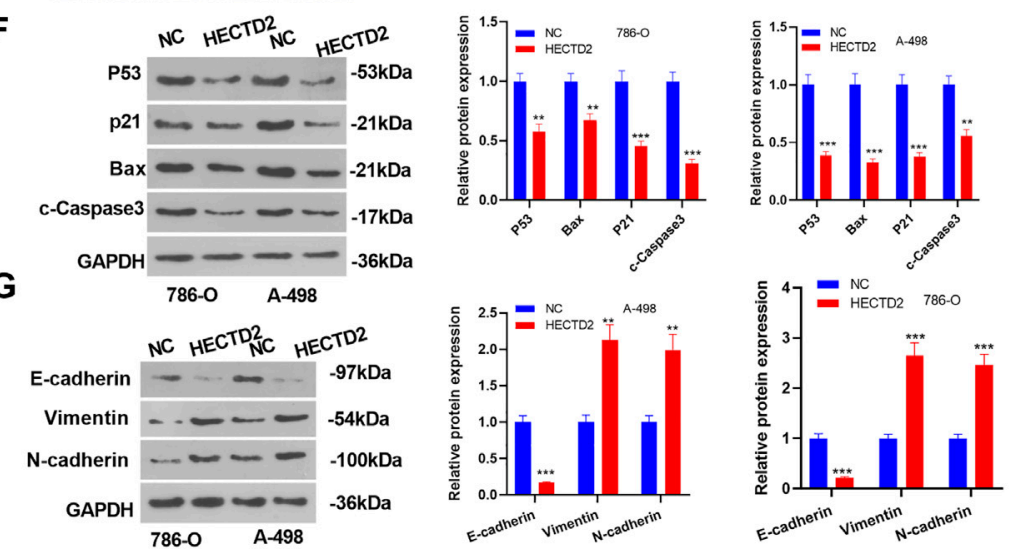

FIGURE 2 | Effect of HECTD2 overexpression on the malignant phenotype of RCC. Overexpression models of HECTD2 were constructed in 786-O and A-498 cell lines. (A): RT-qPCR was implemented to testify the HECTD2 expression. (B-D): RCC cell proliferation and apoptosis were determined by the colony formation experiment, BrdU assay and flow cytometry, respectively. (E) RCC cell migration and invasion were assessed by Transwell assay. (F,G): WB was conducted to examine the expression of P53, Bax, p21, c-Caspase3, E-cadherin, Vimentin, and N-cadherin. ${ }^{\star \star} p<0.01,{ }^{\star \star \star} p<0.001$ vs. NC group. $N=3$. Scale bar $=50 \mu \mathrm{m}$. 


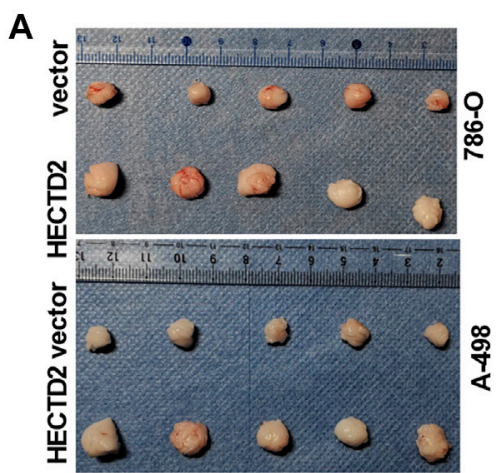

D

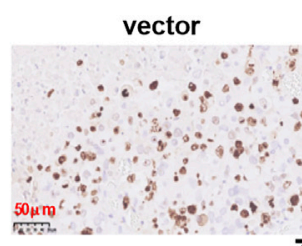

$\mathbf{E}$

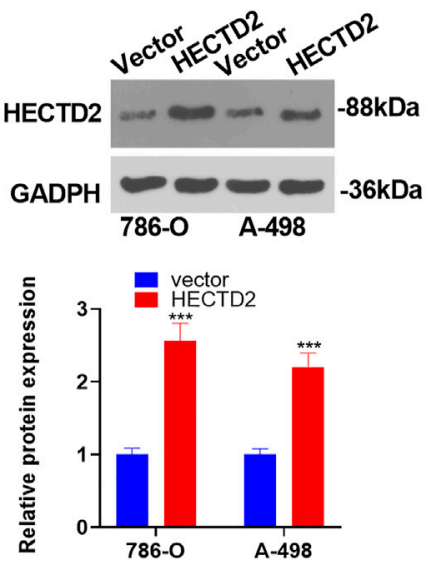

B
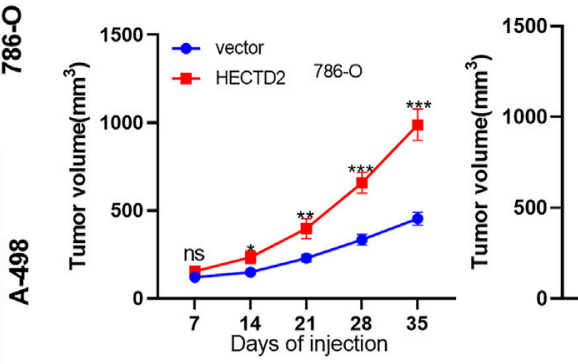

$\rightarrow$ HECTD2

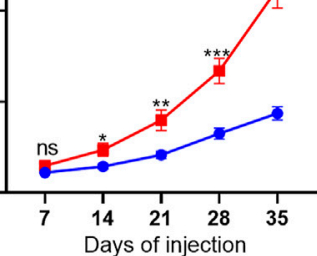

Days of injection
- vector A-498

C
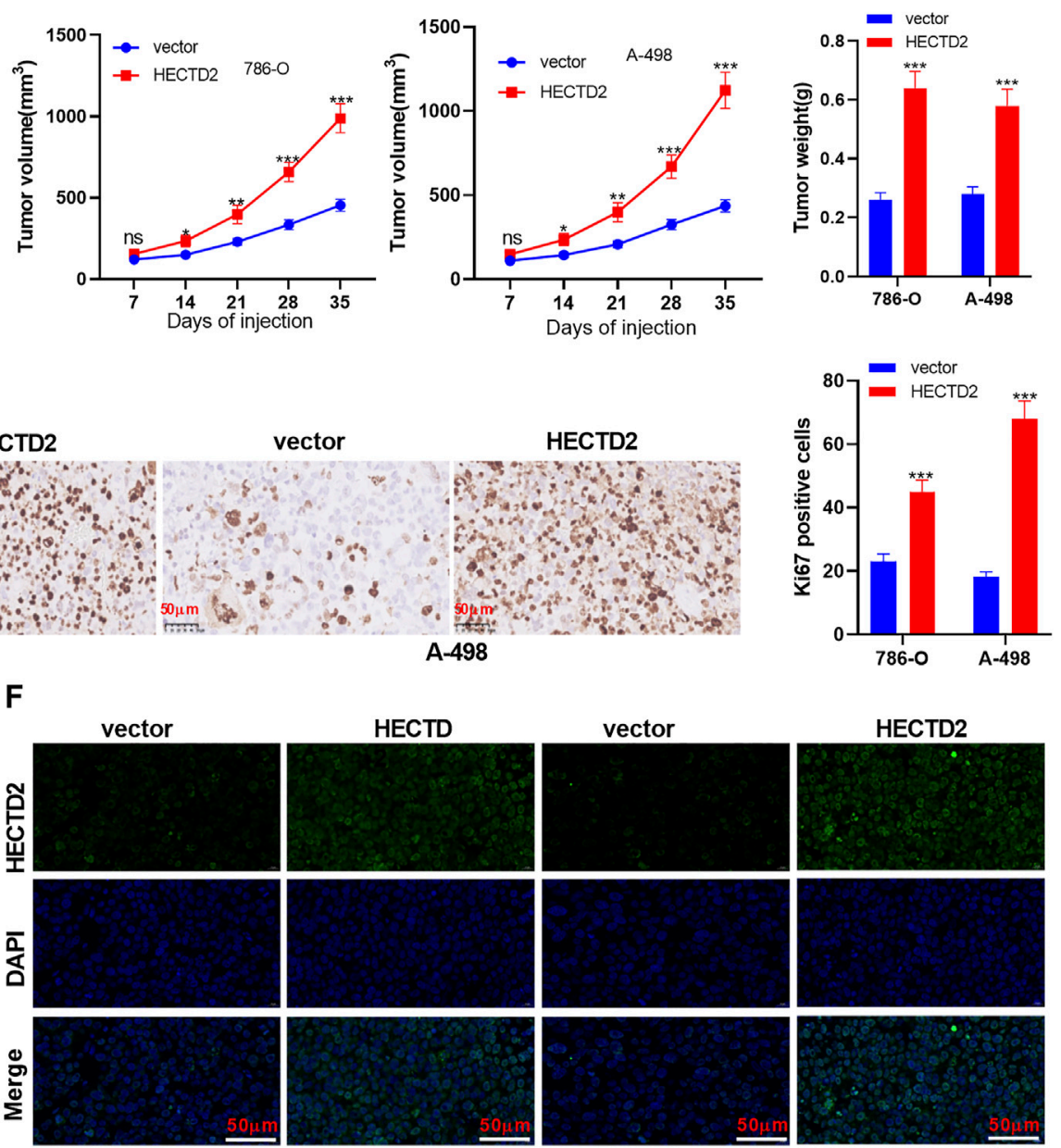

HECTD2

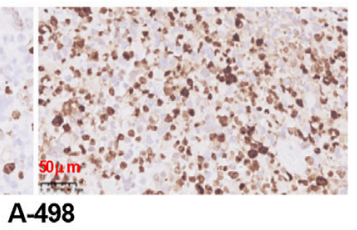

A-498
$\mathbf{F}$

HECTD2
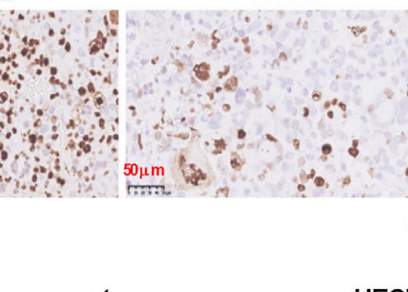
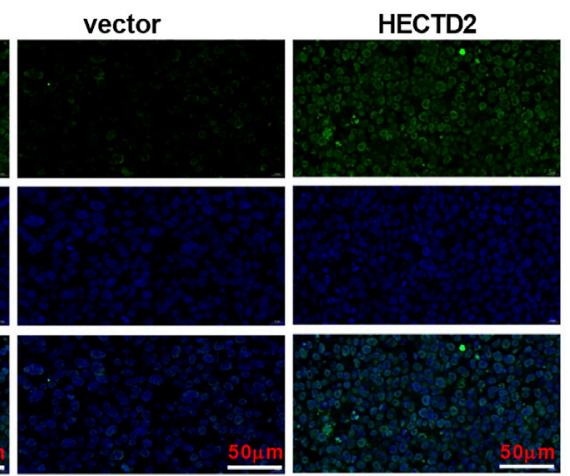

FIGURE 3 | Influences of overexpressing HECTD2 on the malignant phenotypes of RCC in vivo. The HECTD2 overexpression model was established in 786-O and A-498 cells, which were then used for constructing xenografted tumor model in nude mice. The mice were sacrificed at the 35th day after cell transplantation. (A-C): Tumor volume and weight. (D): The number of Ki67-positive cells in 786-O and A-498 cells was evaluated by HC. (E): The profile of HECTD2 in the formed tumor tissues was tested by WB. (F): The fluorescence intensity of HECTD2 was tested by tissue immunofluorescence. ns $p>0.05,{ }^{*} p<0.05,{ }^{* \star} p<0.01,{ }^{\star \star \star} p<0.001 . N=5$. Scale bar $=50 \mu \mathrm{m}$.

expression of HIF-1 $\alpha$ and HECTD2 using RT-qPCR. It turned out that HIF- $1 \alpha$ and HECTD2 were up-regulated after HIF-1 $\alpha$ overexpression. However, compared with the HIF-1 $\alpha$ group, the HIF-1a expression in the HECTD2 group was not changed, while HECTD2 was evidently down-regulated $(p<0.05$, Figure 4A). The colony formation, BrdU assay and flow cytometry were employed to examine RCC cell proliferation and apoptosis. The results unveiled that overexpressing HIF-1a facilitated the proliferation and impeded the apoptosis of RCC. Nevertheless, the findings of the HIF-1 $\alpha+$ si-HECTD2 group were exactly opposite to those of the HIF-1a group $(p<0.05$, Figures 4B-D). Meanwhile, Transwell assay results clarified that HIF-1 $\alpha$ overexpression dramatically strengthened RCC cell invasion and migration. In contrast, RCC cell migration and invasion were significantly hampered in the HIF- $1 \alpha+$ si-HECTD2 group (vs. the HIF-1 1 group) ( $p<0.05$, Figure 4E). WB exhibited that the expression of pro-apoptotic proteins P53, Bax, p21 and c-Caspase 3 were suppressed after overexpressing HIF-1a. However, the profiles of these proteins were heightened in the HIF-1 $\alpha+$ si-HECTD2 group (vs. the HIF-1 1 group) $(p<$ 0.05 , Figure 4F). Also, WB results confirmed that HIF-1 $\alpha$ overexpression significantly hampered the expression of E-cadherin and elevated the levels of Vimentin and $\mathrm{N}$-cadherin (vs. the control group). The addition of siHECTD2 to HIF-1a subverted the effect of HIF-1 $\alpha(p<0.05$, 
A

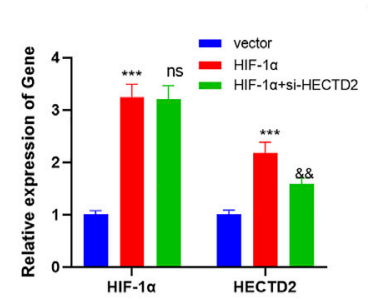

B

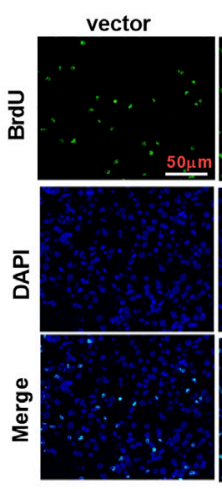

HIF-1 $\alpha$
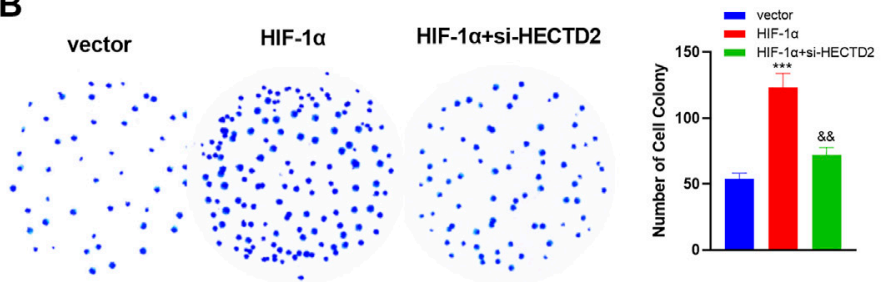

D

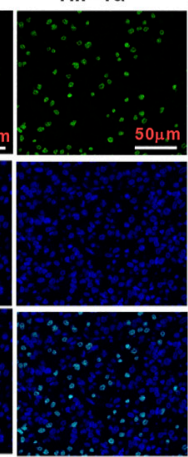

HIF-1 $\alpha+$ Si-HECTD2

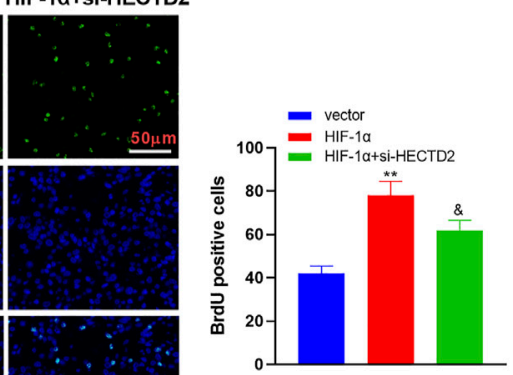

vector
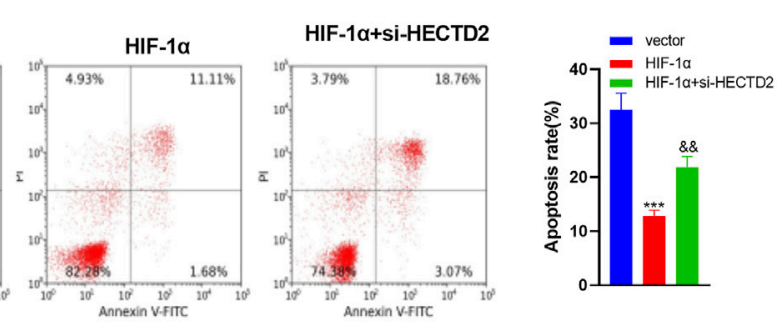

E

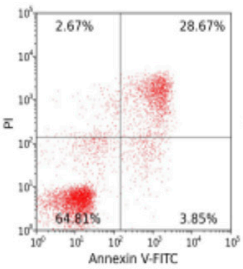

HIF-1 $\alpha$

HIF-1 $\alpha+$ si-HECTD2
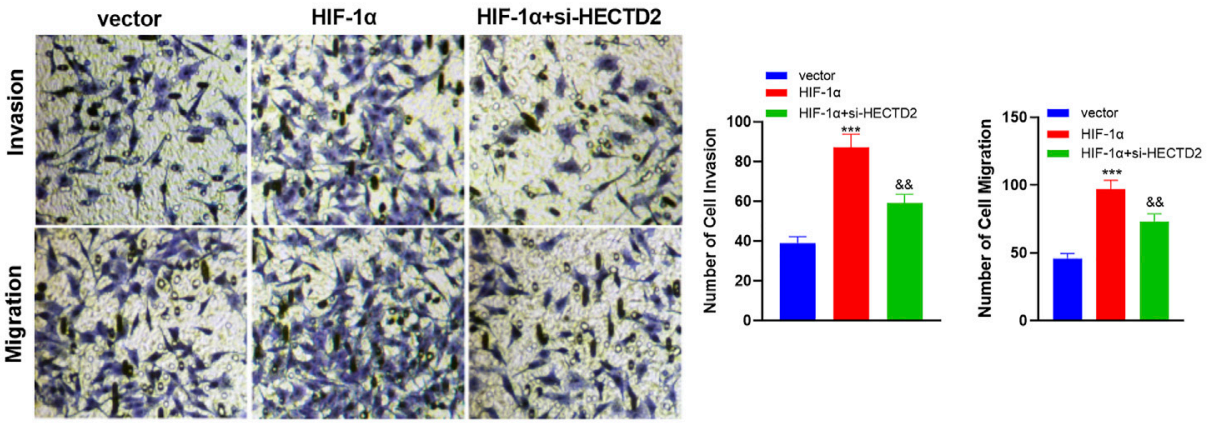

F

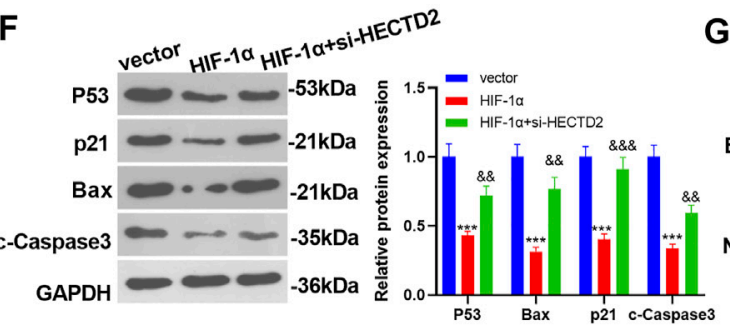

G

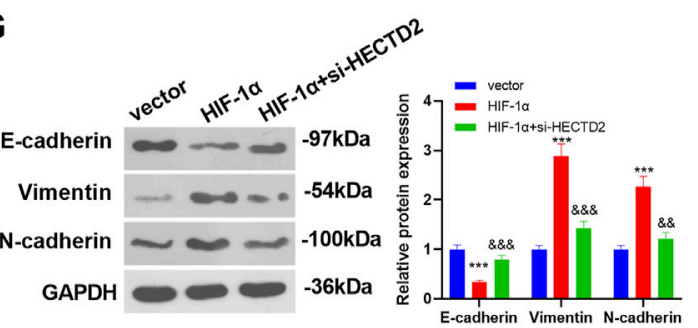

FIGURE 4 | HIF-1a heightened the expression of HECTD2 and its malignant phenotype. HECTD2 was knocked down in 786-O cells overexpressing HIF-1a. (A): RT-qPCR was carried out to monitor the levels of HIF-1 a and HECTD2. (B-D): RCC cell proliferation and apoptosis were checked using the colony formation experiment, BrdU assay and flow cytometry, respectively. (E): Transwell assay was conducted to measure RCC cell migration and invasion. (F,G): The expression of P53, Bax, p21, C-Caspase3, E-cadherin, Vimentin, and N-cadherin was compared by WB. ${ }^{* \star *} p<0.001$ (vs. vector group). ns $p>0.05, \& \& p<0.01$ (vs. HIF-1a group). N=3. Scale bar $=50 \mu \mathrm{m}$. 


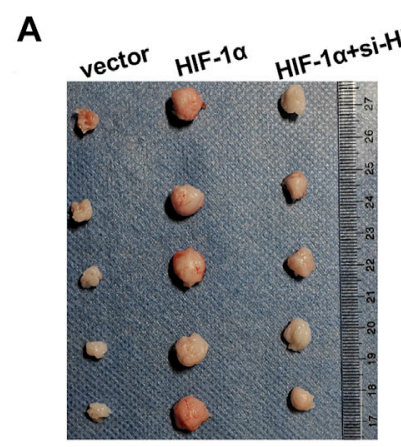

D
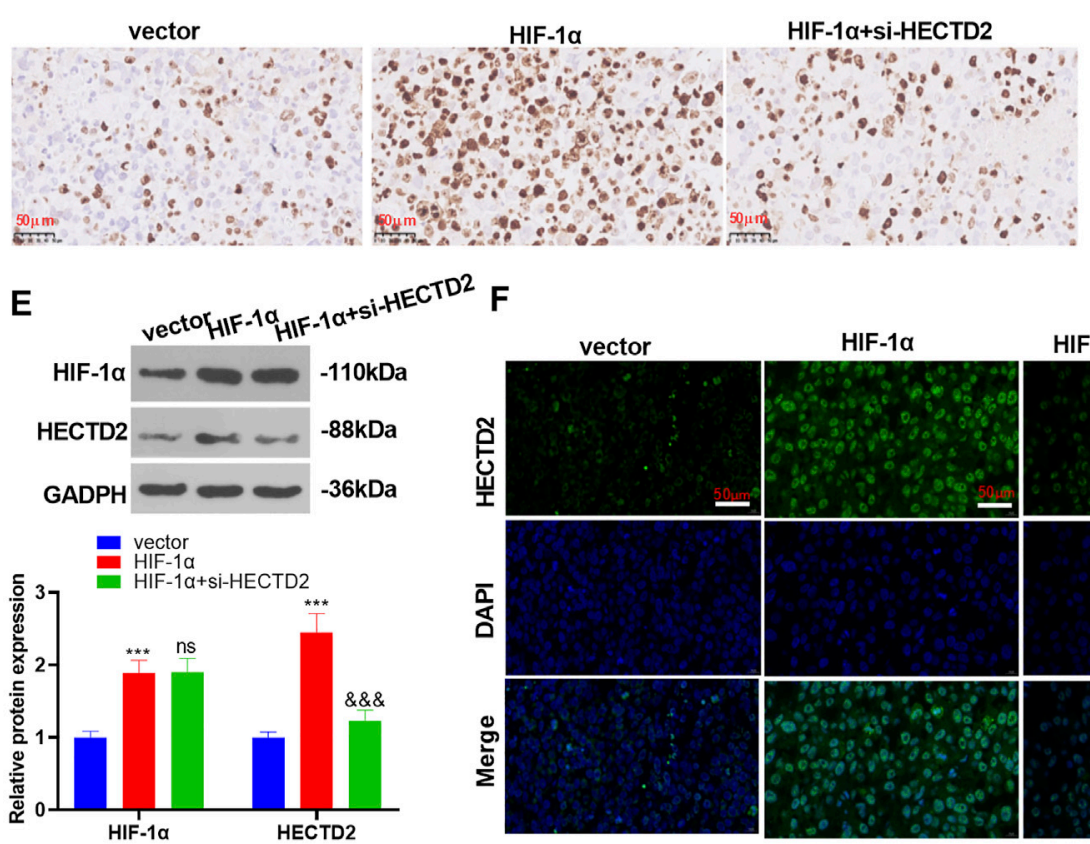

B

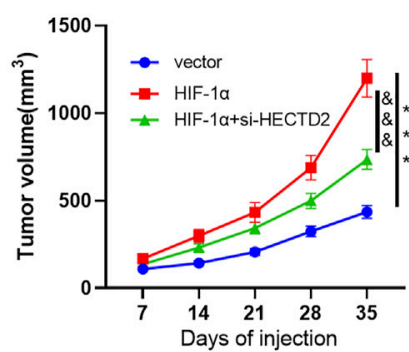

C

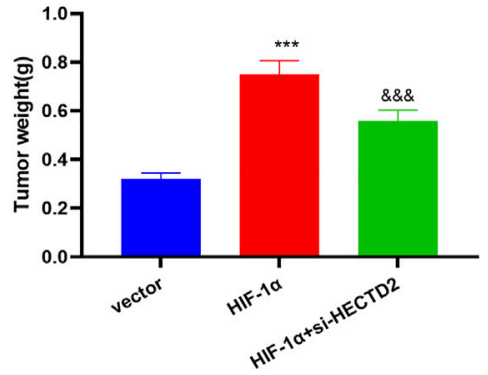

G

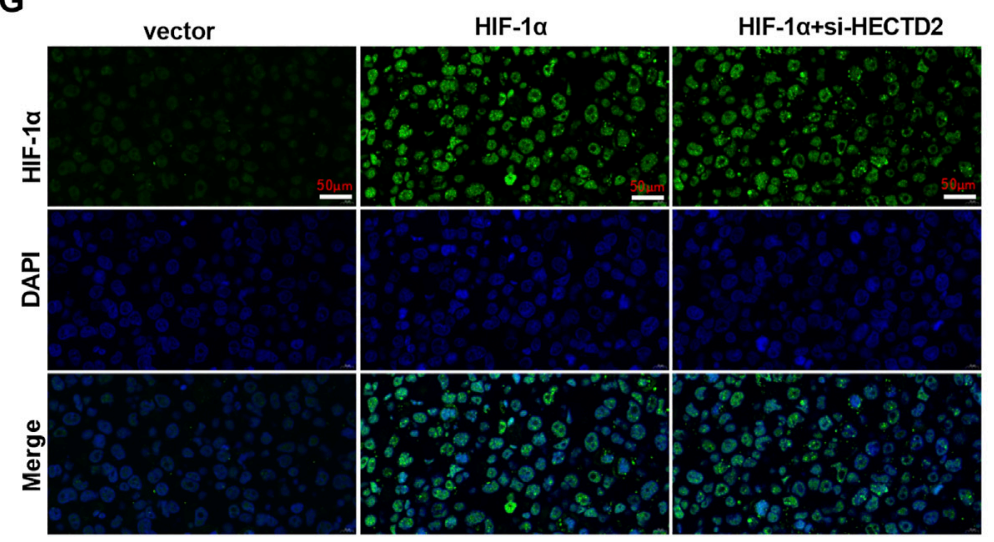

FIGURE 5 | Impacts of the HIF-1a/HECTD2 axis on RCC cell growth in vivo. HIF-1a overexpression and/or HECTD2 knockdown cell model was used for constructing xenografted tumor model in nude mice. The mice were sacrificed at the 35th day after cell transplantation. (A-C): Tumor volume and weight. (D): IHC was adopted to count the number of Ki67-positive cells in 786-O and A-498. (E): The profiles of HIF-1a and HECTD2 in 786-O and HECTD2 cells were monitored by WB. $(\mathbf{F}, \mathbf{G})$ : The fluorescence intensity of HIF-1a and HECTD2 was checked by tissue immunofluorescence. ns $p>0.05,{ }^{\star} p<0.05,{ }^{* *} p<0.01,{ }^{* \star *} p<0.001 . N=5$. Scale bar $=50 \mu \mathrm{m}$. 
A

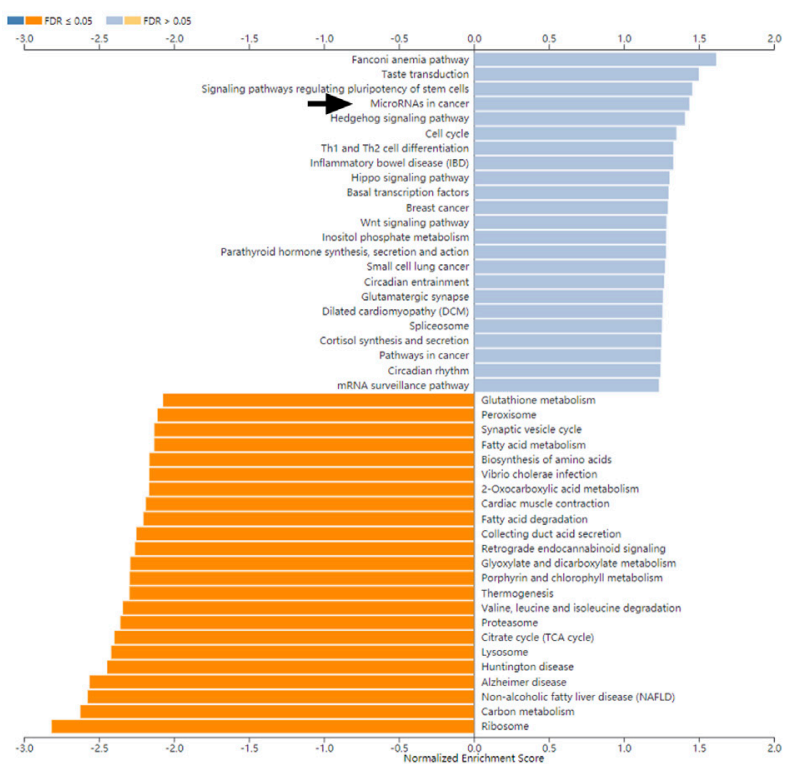

B

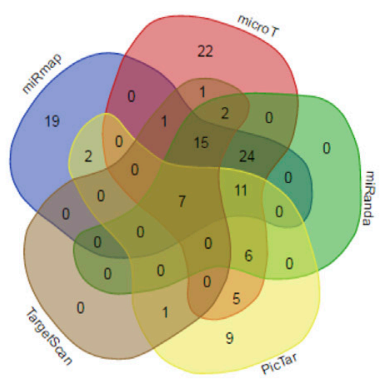

D

HECTD2-WT: 5'uuuagaUGUGUACACAGCUUUu 3' miR-320a: $3^{\prime}$ agcgggÁGÁGUU'GGGUCGAAÁa 5' HECTD2-MT: 5 unuagaUUGgGUGAUAGCCAUUU I 3
C

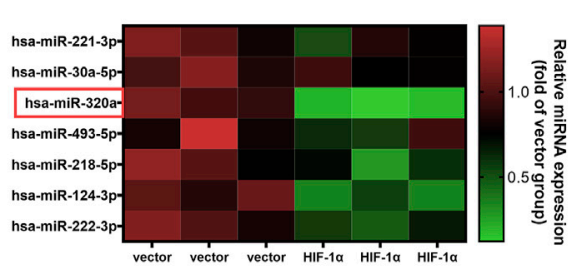

E

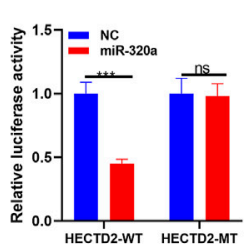

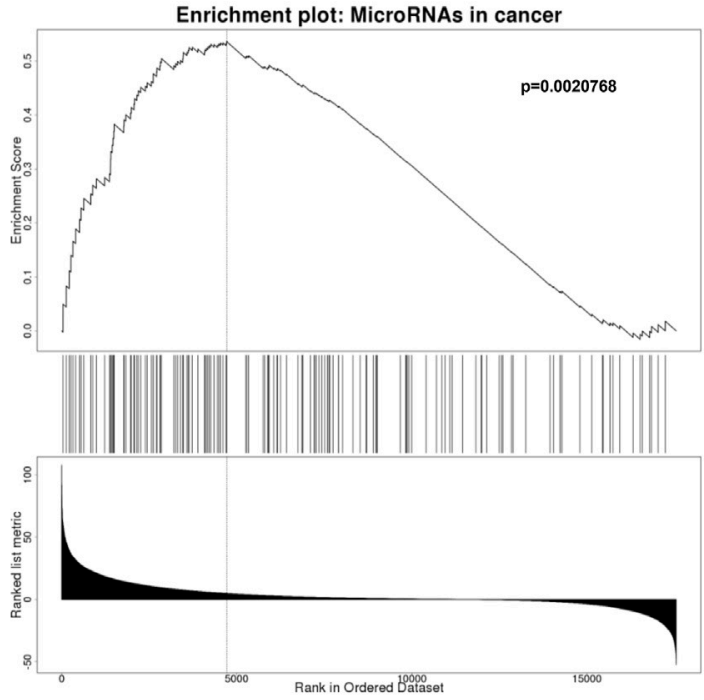

Enrichment plot: MicroRNAs in cancer

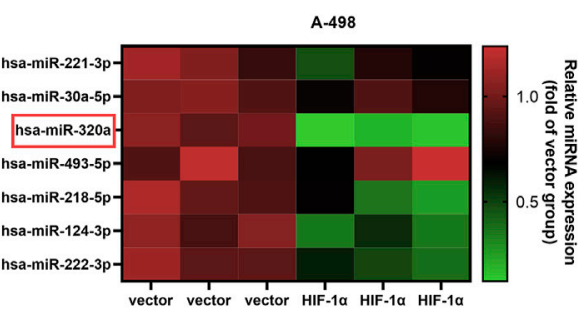

G

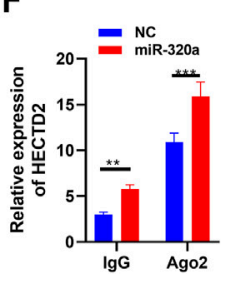

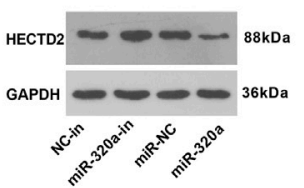

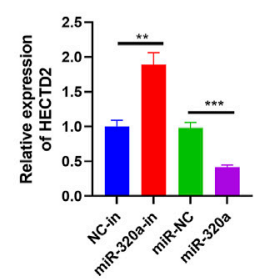

FIGURE 6 | miR-320a targeted HECTD2. (A) The positive or negative related genes of HECTD2 in TGCA database was analyzed via LinkedOmics, and those genes were subjected to GSEA enrichment analysis. HECTD2 is potentially involved in a KEGG pathway of MicroRNAs in Cancer. (B): Targetscan, miRmap, microT, PicTar and miRanda databases were utilized to search the miRNA target of HECTD2. Venn diagram was utilized to analyze the common target miRNAs of HECTD2. Seven common miRNAs were identified, including has-miR-221-3p, has-miR-30a-5p, has-miR-320a, has-miR-493-5p, has-miR-218-5p, has-miR-124-3p and has-miR-222-3p. (C): RT-PCR was used for determining the seven miRNAs in 786-O and A-498 cells transfected with HIF-1 a. (D): HECTD2 contained the binding sites with miR-320a. (E,F). Dual-luciferase reporter assay and RIP assay were applied to probe the binding of miR-320a to HECTD2. G: HECTD2 level in 786-O cells transfected with miR-320a mimics or miR-320a inhibitors was tested by WB. NS $p>0.05,{ }^{* *} p<0.01,{ }^{* * *} p<0.001 . N=3$.

Figure 4G). These results manifested that up-regulating HIF-1a in 786-O cells resulted in the elevation of HECTD2 profiles and the aggravation of RCC.

\section{Effects of the HIF-1 $\alpha$ /HECTD2 Axis on RCC Cell Growth in vivo}

To further validate the impact of the HIF-1 $\alpha$ /HECTD2 axis on tumor growth in vivo, we established HIF-1a overexpression and/or HECTD2 knockdown models in 786-O cells and discovered that overexpressing HIF-1a increased tumor volume and weight (vs. the vector group). However, HECTD2 knockdown suppressed RCC tumor volume and weight (vs. the HIF-1a group) ( $p<0.05$, Figures 5A-C). Also, immunohistochemical results exhibited evident facilitation in the Ki67-positive cell number in 786-O cells after HIF-1a overexpression, whereas the effect was reversed after HECTD2 knockdown ( $p<0.05$, Figure 5D). Besides, WB results illustrated that the profiles of HIF-1 $\alpha$ and HECTD2 were elevated after HIF-1a overexpression in 786-O cells. However, compared with the HIF-1a group, the HIF-1a expression remained unchanged in the HIF-1a + si-HECTD2 group, but 
A

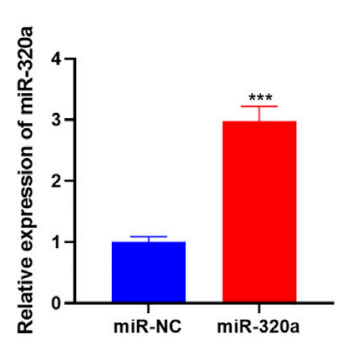

B

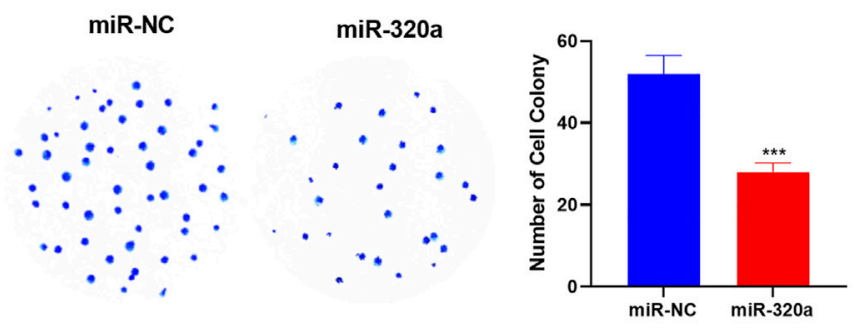

C
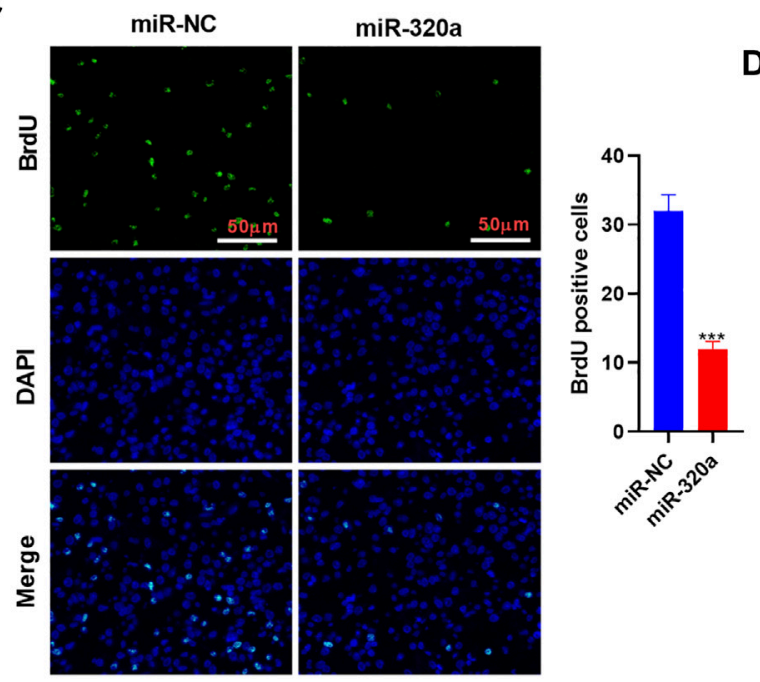

D
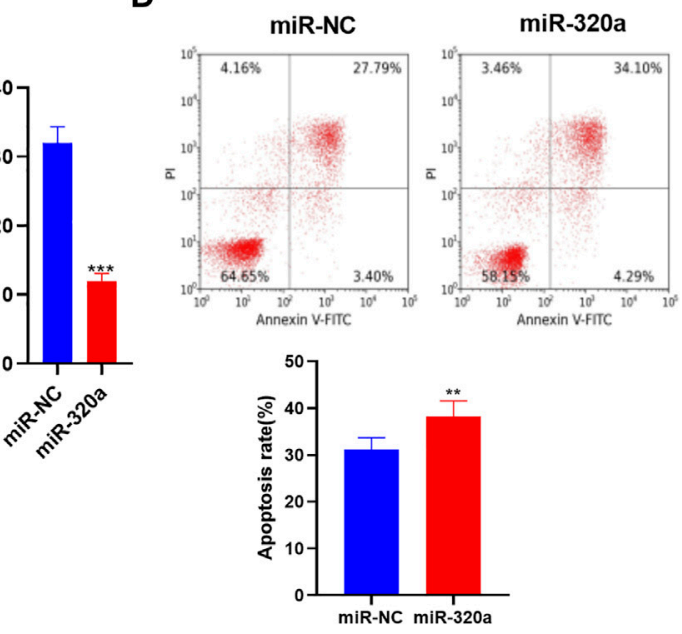

E
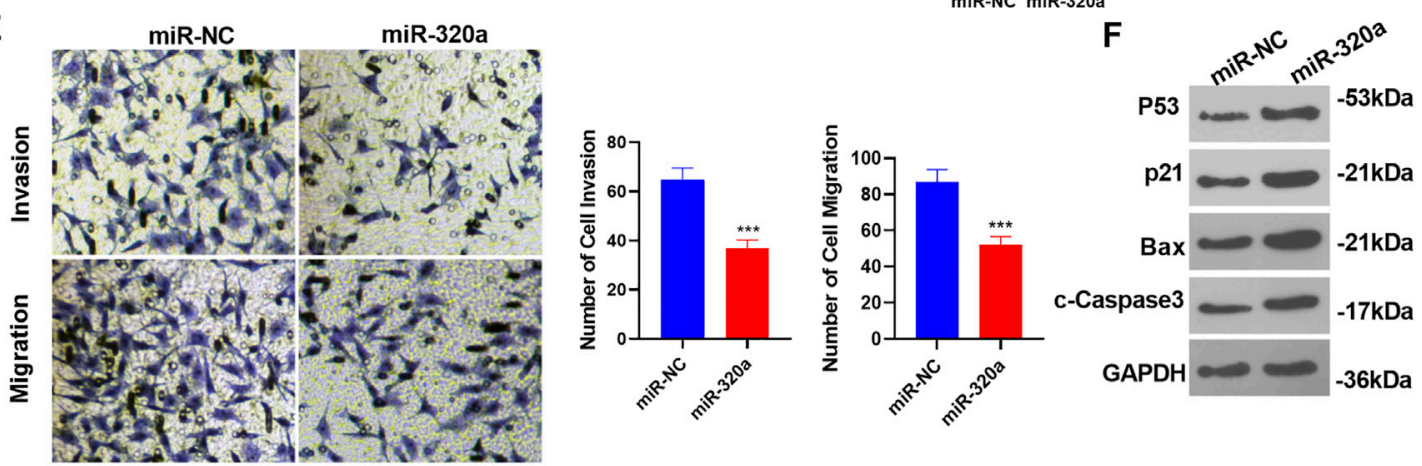

G

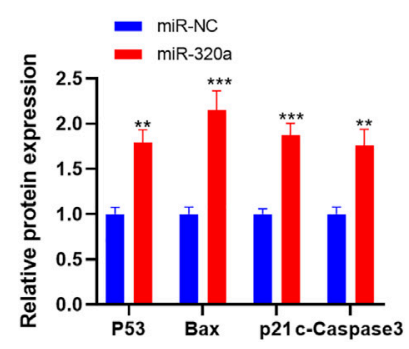

H

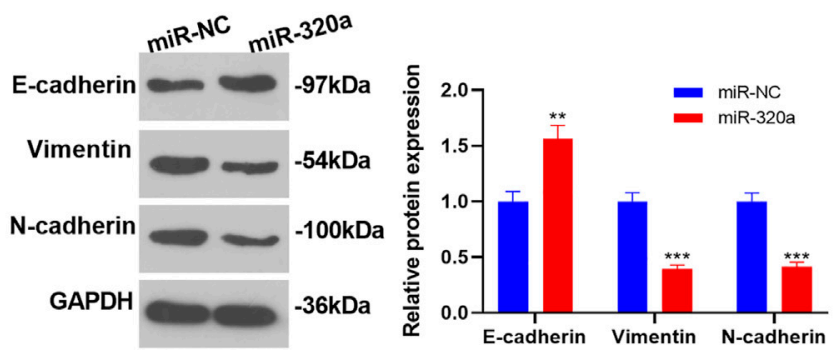

FIGURE 7 | Influences of miR-320a on the malignant phenotype in RCC. miR-320a mimics were transfected into A-498 cells. (A): The miR-320 profile was determined by RT-qPCR. (B-D): RCC cell proliferation and apoptosis were checked by the colony formation assay, BrdU assay and flow cytometry, respectively. (E): Transwell assay was implemented to verify RCC cell migration and invasion. (F-H): WB was conducted to evaluate the expression of P53, Bax, p21, C-Caspase3, E-cadherin, Vimentin, and N-cadherin. ${ }^{* *} p<0.01,{ }^{* * *} p<0.001 . N=3$. 
A

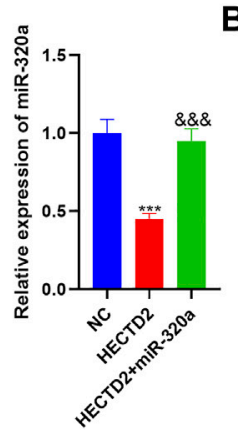

B

C
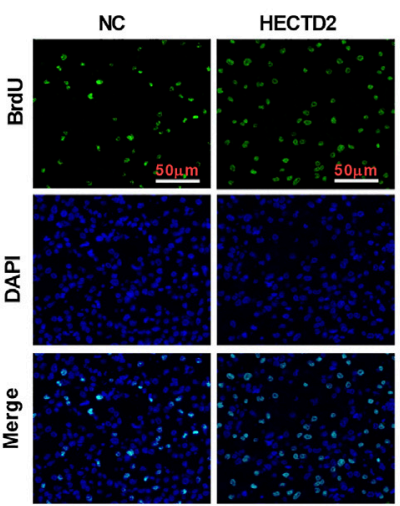

D
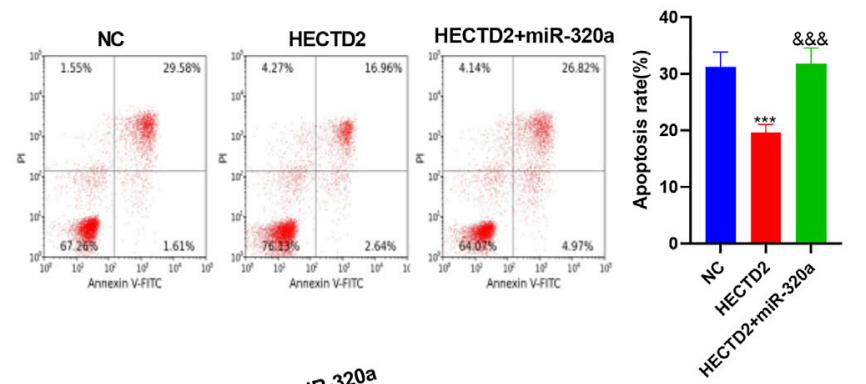

HECTD2+miR-320a
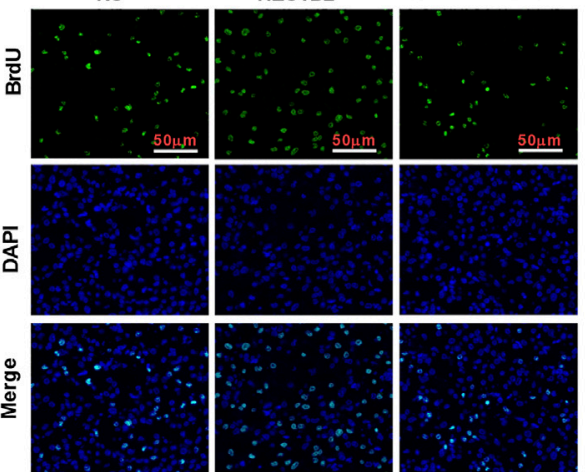

F
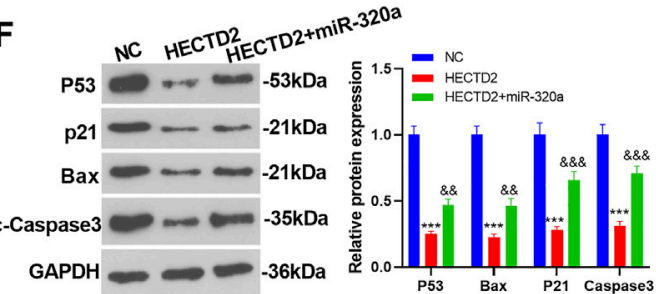

E

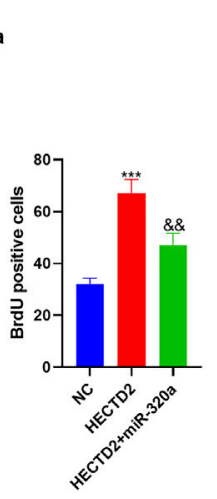

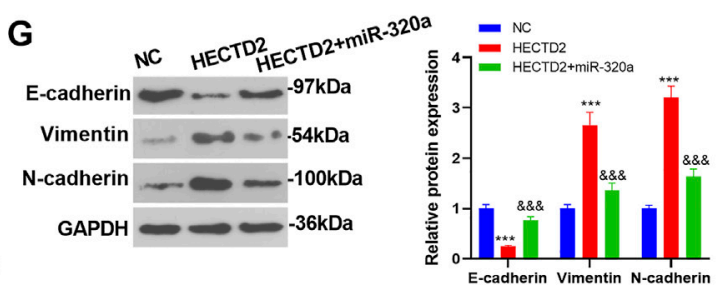
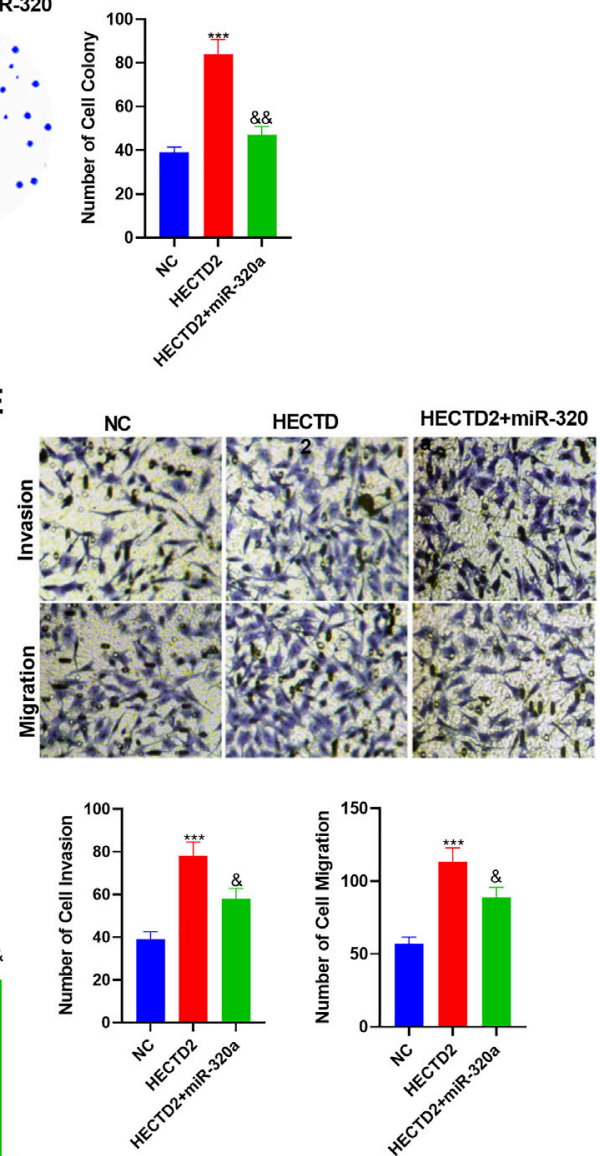

FIGURE 8 | miR-320a affected RCC growth and EMT via HECTD2. Transfection of miR-320a mimics was made in 786-O cells with overexpressed HECTD2. (A). RT-qPCR was implemented to examine miR-320 expression. (B-D): The colony formation assay, BrdU assay, and flow cytometry were applied to assess RCC cell proliferation and apoptosis. RCC cell migration and invasion were tested by Transwell assay. (F,G): The profiles of P53, Bax, p21, C-Caspase3, E-cadherin, Vimentin, and $\mathrm{N}$-cadherin were compared by Transwell assay. ${ }^{\star \star \star} p<0.001, \& p<0.05, \& \& p<0.01, \& \& \& p<0.001 . N=3$.

HECTD2 was significantly down-regulated $(p<0.05$, Figure 5E). The results of tissue immunofluorescence exhibited that the fluorescence intensity of HIF-1a and HECTD2 was more pronounced in the HIF-1a group (vs. the vector group). Nevertheless, compared with the HIF-1 $\alpha$ group, the fluorescence intensity of HIF-1a in the HIF-1a + siHECTD2 group showed little change, but the fluorescence intensity of HECTD2 was dramatically weakened $(p<0.05$,
Figures 5 F,G). These findings further confirmed the influence of HIF-1a overexpression on HECTD2 and RCC cell growth in vivo.

\section{miR-320a Targeted HECTD2}

For analyzing the upstream mediator of HECTD2, we analyzed the positive or negative related genes of HECTD2 via LinkedOmics, and those genes were subjected to GSEA 
enrichment analysis. The results indicated that HECTD2 is involved in a KEGG pathway of MicroRNAs in Cancer (Figure 6A). Inspired by the miRNA-mRNA regulatory network, we adopted Targetscan, miRmap, microT, PicTar and miRanda to search the miRNA target of HECTD2 and discovered that HETCD2 had seven miRNAs as its common targets (Figure 7A). After that, we examined the profiles of these seven miRNAs in HIF-1 $\alpha$ overexpressing cells by RT-PCR. It turned out that miR-320a was most significantly down-regulated (Figure 6C). The base binding sites between miR-320a and HECTD2 was shown in Figure 6D. The dual-luciferase reporter assay results demonstrated that miR-320a weakened the luciferase activity of HECTD2-WT, but it had no substantial effect on HECTD2-MUT ( $p<0.05$, Figure 6E). The RIP results demonstrated that miR-320a mimic transfection resulted in a higher amount of HECTD2 deposited in the Ago2 antibody group than that in the IgG group, implying that HECTD2 was bound to Ago2 via miR320a $(p<0.05$, Figure 6F). Moreover, the effect of modulating miR-320a on HECTD2 expression was examined by RT-qPCR. Interestingly, overexpressing miR-320a hampered the HECTD2 profile, while miR-320a knockdown showed the reverse $(p<0.05$, Figure 6G). These findings testified that there was a targeting correlation between miR-320a and HECTD2.

\section{Impacts of miR-320a on the Malignant Phenotypes of RCC}

To figure out the influences of miR-320a on HECTD2 and RCC evolvement, we transfected miR-320a mimics in A-498 cells and verified the transfection validity by RT-qPCR $(p<$ 0.05, Figure 7A). Meanwhile, the results of the colony formation assay, BrdU assay and flow cytometry illustrated that overexpressing miR-320a restrained RCC cell proliferation and intensified apoptosis $(p<0.05$, Figures 7B-D). Additionally, Transwell assay results manifested that RCC cell migration and invasion were curbed by miR320 a overexpression $(p<0.05$, Figure 7E). Besides, WB results uncovered that the transfection of miR-320a mimics upregulated P53, Bax, $\mathrm{p} 21$, and $\mathrm{c}$-Caspase3 (vs. miR-NC group) ( $p<0.05$, Figures 7F,G). Moreover, WB results also signified that overexpressing miR-320a significantly facilitated E-cadherin expression and hampered the levels of Vimentin and $\mathrm{N}$-cadherin (vs. the miR-NC group) $(p<$ 0.05 , Figure $7 \mathbf{H})$. These results testified that transfection of miR-320a mimics in A-498 cells alleviated RCC progression.

\section{miR-320a Influenced RCC Growth and Epithelial-Mesenchymal Transition via HECTD2}

To explore the specific mechanism by which miR-320a affected RCC growth and EMT, we transfected miR-320a mimics in 786$\mathrm{O}$ cells overexpressing HECTD2 and assayed the transfection validity by applying RT-qPCR. As a result, compared with the NC group, miR-320a was down-regulated in the HECTD2 group and was up-regulated in the miR-320a group ( $p<0.05$, Figure 8A).
The colony formation assay, BrdU assay and flow cytometry results exhibited enhanced RCC cell proliferation and significantly repressed apoptosis in the HECTD2 group compared to the NC group, while the transfection of miR320a mimics exerted the opposite function $(p<0.05$, Figures 8B-D). Meanwhile, Transwell assay results showed that RCC migration and invasion were significantly enhanced in the HECTD2 group compared with the NC group, while they were attenuated after the miR-320a mimic transfection $(p<$ 0.05 , Figure 8E). Also, WB exhibited that the protein profiles of P53, Bax, p21 and c-Caspase 3 were evidently declined in the HECTD2 group (vs. the NC group). Nevertheless, these proteins were significantly up-regulated in the HECTD2 +miR-320a group (vs. the HECTD2 group) ( $p<0.05$, Figure $8 \mathbf{8 F}$ ). Moreover, WB results illustrated that the E-cadherin profile was impeded, while Vimentin and N-cadherin were significantly up-regulated in the HECTD2 group (vs. the NC group). The HECTD2+miR-320a group had completely opposite results to the HECTD2 group ( $p<0.05$, Figure 8G). These findings manifested that HECTD2 enhanced RCC cell growth and EMT in 786-O cells, but transfection with miR-320a mimics significantly alleviated RCC (Graphical Abstract).

\section{DISCUSSION}

RCC is among the most familiar tumors of the genitourinary system. Therefore, it's urgent to research the carcinogenesis and progressive mechanism of RCC and to search for potential molecular biomarkers and therapeutic targets (Chow et al., 2010). Here, we discovered that HIF-1a and HECTD2 were enhanced in RCC, and in-vivo studies testified that overexpressing HECTD2 and HIF-1a hampered apoptosis and induced tumor growth of RCC cells. Further studies illustrated that HIF-1a contributed to RCC progression by inhibition of miR-320a, an upstream regulator of HECTD2.

A major character of cancer environment, hypoxia is often caused by abnormal tumor growth when tumor cells are lack of oxygen supply (Jahanban-Esfahlan et al., 2018). HIF-1a is not only a key nuclear transcription factor in the tumor hypoxia, but also an upstream transcriptional regulator of genes associated with tumor cell proliferation, apoptosis, neovascularization, invasion and metastasis (Lin et al., 2017; Knight and Stanley, 2019). Emerging researches have reported that HIF-1a overexpression contributes to poor prognosis and is a therapy target in diversified cancers (Yang et al., 2014; Egawa et al., 2021; Yang et al., 2021). Many strategies have been used in restraining tumor progression and chemoresistance by targeting HIF-1a (Luo et al., 2021). In RCC, HIF-1 $\alpha$ upregulation enhances proliferation, migration and survival by mediating metabolic reprogramming (de Carvalho et al., 2021). Transglutaminase 2 (TGase 2) promoted angiogenesis by inducing p53 degradation, thus activating HIF-1a/VEGF2R pathway (Lee et al., 2020). In addition, HIF- $1 \alpha$ has a role in regulating RCC cell glycolysis. HIF$2 a$, another Hypoxia-inducible factor, affects antigen presentation, interferon signalling and CD8 T cell infiltration and activation (Hoefflin et al., 2020). Presently, we discovered 
that HIF-1a was up-regulated in RCC tissues and induces enhanced RCC cell proliferation, growth, migration and reduced apoptosis.

As a vital miRNA, miR-320a has been proved to have significant values in colorectal cancer (Zhang et al., 2021a), epithelial ovarian cancer (Zhang et al., 2021b), nasopharyngeal cancer (Xing et al., 2021), melanoma (Fu et al., 2020). More importantly, it has been reported that various miRNAs, such as miR-155 (Meng et al., 2021), miR-224-5p (Qin et al., 2021), miR30b-5p (Zhang et al., 2021c), etc., are involved in the development of RCC by targeting different genes. Interestingly, miR-320a has been found downregulated in RCC tissues and cell lines (data from TCGA), and it represses the proliferation, invasion via targeting FoxM1 (Zhao et al., 2018). This article revealed that overexpressing miR-320a hampered RCC proliferation, migration, invasion, and EMT and facilitated apoptosis, confirming that miR-320a was involved in RCC evolvement as a tumor suppressor.

miRNAs are dysregulated in cancers under hypoxia microenvironment, which are particularly associated with poor prognosis in cancer patients (Yang et al., 2020). Those miRNAs could be regulated at transcriptional and post-translational levels (Bandara et al., 2017). Recently, studies have revealed that hypoxia-stimulated long non coding RNAs (lncRNAs) inhibit miRNAs in cytoplasm and represses the expression of the later (Li et al., 2021; Wan et al., 2021). Moreover, hypoxia promotes the released of extracellular vesicles (EVs), which are enriched with miRNAs, thus inducing apoptosis decrease and proliferation enhancement (Yin et al., 2021; Endzeliņš et al., 2018). In RCC, hypoxia induced upregulation of miR-346 and N-myc downstream-regulated gene 2 (NDRG2) inhibition in renal cancer cells. miR-346 represses NDRG2 and promotes the malignant behaviors of RCC cells (Su et al., 2020). As hypoxia-related transcription factors, HIF-1 and HIF-2 have been confirmed to play a dominant role in transcriptional gene regulation in hypoxia, including miRNAs (Serocki et al., 2018), and this mechanism is also involved in RCC progression (White and Yousef, 2010; Rustum et al., 2018). In this study, we found that HIF-1a overexpression significantly inhibits miR-320a expression, which suggests that HIF-1a potentially induces increased malignant phenotypes of RCC cells by suppressing miR-320a.

HECTD family members have been found associated with hypoxia microenvironment alteration. For instance, Hectd1 is regulated by insulin, heat, and hypoxia during embryogenesis in mice (D'Alonzo et al., 2019). Hypothermic oxygenated perfusion (HOP) prevents ischemia-reperfusion-mediated liver damage. The underlying mechanism revealed that both the DOC and HECT domains of HECTD3 induce polyubiquitination of TRAF3 at Lys138, and ubiquitinated TRAF3 promotes oxidative stress and inflammation (Zhou et al., 2021). Here, we showed that
HECTD2 upregulation enhances RCC progression both in vitro and in vivo, and HIF-1 $\alpha$ had a positives relationship with HECTD2 and increased its expression. To further clarify the mechanism of HECTD2, we searched its upstream target miRNAs and found a binding site shared by miR-320a and HECTD2. Furthermore, the dual-luciferase and RIP experiments confirmed the targeted binding association between the two. As a matter of fact, HECTD2 has been reported to exert a prominent role in tumor and is modulated by miRNA (Sun et al., 2014). What's more, transfection of miR-320a mimics dampens RCC cells proliferation, migration, invasion followed by HECTD2 upregulation. These results showed that miR-320a affected RCC progression via directly targeting HECTD2.

Overall, our study confirmed that 1) HECTD2 is upregulated in RCC and aggravates RCC progression; 2) miR320 a functions as a tumor suppressor in RCC by targeting HECTD2; 3) HIF-1a induces HECTD2 upregulation by repressing miR-320a. In summary, this study manifests a novel regulatory axis of HIF-1a/miR-320a/HECTD2 in RCC progression, which may be a viable therapeutic target for RCC. However, further experiments are needed for investigating the potential HIF-1a/miR-320a/HECTD2 axis in treating RCC in vivo and the downstream mechanism of HECTD2 in RCC.

\section{DATA AVAILABILITY STATEMENT}

The original contributions presented in the study are included in the article/Supplementary Material, further inquiries can be directed to the corresponding authors.

\section{ETHICS STATEMENT}

The studies involving human participants were reviewed and approved by the Animal Ethics committee of Sichuan Provincial Peopl's Hospital. The patients/participants provided their written informed consent to participate in this study.

\section{AUTHOR CONTRIBUTIONS}

Conceived and designed the experiments: YX and ZM; Performed the experiments: DL and TS; Statistical analysis: JY and QY; Wrote the paper: DL and TS. All authors read and approved the final manuscript.

\section{FUNDING}

This work was supported by Sichuan Provincial Commission of Health and Family Planning (16PJ437). 


\section{REFERENCES}

Bandara, K. V., Michael, M. Z., and Gleadle, J. M. (2017). MicroRNA Biogenesis in Hypoxia. Microrna 6 (2), 80-96. doi:10.2174/2211536606666170313114821

Cheng, C., Guo, L., Ma, Y., Wang, Z., Fan, X., and Shan, Z. (2020). Up-Regulation of miR-26a-5p Inhibits E2F7 to Regulate the Progression of Renal Carcinoma Cells. Cancer Manag. Res. 12, 11723-11733. doi:10.2147/CMAR.S271710

Chow, W.-H., Dong, L. M., and Devesa, S. S. (2010). Epidemiology and Risk Factors for Kidney Cancer. Nat. Rev. Urol. 7 (5), 245-257. doi:10.1038/ nrurol.2010.46

Coon, T. A., McKelvey, A. C., Lear, T., Rajbhandari, S., Dunn, S. R., Connelly, W., et al. (2015). The Proinflammatory Role of HECTD2 in Innate Immunity and Experimental Lung Injury. Sci. Transl. Med. 7 (295), 295ra109. doi:10.1126/ scitranslmed.aab3881

D’Alonzo, D., Emch, F. H., Shen, X., Bruder, E., De Geyter, C., and Zhang, H. (2019). Hectd1 Is Essential for Embryogenesis in Mice. Gene Expr. Patterns 34, 119064. doi:10.1016/j.gep.2019.119064

de Carvalho, P. A., Bonatelli, M., Cordeiro, M. D., Coelho, R. F., Reis, S., Srougi, M., et al. (2021). MCT1 Expression Is Independently Related to Shorter Cancerspecific Survival in clear Cell Renal Cell Carcinoma. Carcinogenesis, bgab100. Advance online publication. doi:10.1093/carcin/bgab100

Di Franco, G., Palmeri, M., Sbrana, A., Gianardi, D., Furbetta, N., Guadagni, S., et al. (2020). Renal Cell Carcinoma: The Role of Radical Surgery on Different Patterns of Local or Distant Recurrence. Surg. Oncol. 35, 106-113. doi:10.1016/ j.suronc.2020.08.002

Egawa, N., Tanaka, T., Matsufuji, S., Yamada, K., Ito, K., Kitagawa, H., et al. (2021). Antitumor Effects of Low-Dose Tipifarnib on the mTOR Signaling Pathway and Reactive Oxygen Species Production in HIF-1 $\alpha$-Expressing Gastric Cancer Cells. FEBS Open Bio 11, 1465-1475. doi:10.1002/2211-5463.13154

Endzelinšš, E., Ābols, A., Bušs, A., Zandberga, E., Palviainen, M., Siljander, P., et al. (2018). Extracellular Vesicles Derived from Hypoxic Colorectal Cancer Cells Confer Metastatic Phenotype to Non-Metastatic Cancer Cells. Anticancer Res. 38 (9), 5139-5147. doi:10.21873/anticanres.12836

Ferragut Cardoso, A. P., Udoh, K. T., and States, J. C. (2020). Arsenic-Induced Changes in miRNA Expression in Cancer and Other Diseases. Toxicol. Appl. Pharmacol. 409, 115306. doi:10.1016/j.taap.2020.115306

Fu, G., Lu, J., Zheng, Y., Wang, P., and Shen, Q. (2020). MiR-320a Inhibits Malignant Phenotype of Melanoma Cells via Targeting PBX3. J. BUON 25 (4), 2071-2077.

Ge, X., Pan, M.-H., Wang, L., Li, W., Jiang, C., He, J., et al. (2018). HypoxiaMediated Mitochondria Apoptosis Inhibition Induces Temozolomide Treatment Resistance through miR-26a/Bad/Bax axis. Cell Death Dis 9 (11), 1128. doi:10.1038/s41419-018-1176-7

Gudas, L. J., Fu, L., Minton, D. R., Mongan, N. P., and Nanus, D. M. (2014). The Role of HIFla in Renal Cell Carcinoma Tumorigenesis. J. Mol. Med. 92 (8), 825-836. doi:10.1007/s00109-014-1180-z

Hoefflin, R., Harlander, S., Schäfer, S., Metzger, P., Kuo, F., Schönenberger, D., et al. (2020). HIF-1 $\alpha$ and HIF-2 $\alpha$ Differently Regulate Tumour Development and Inflammation of clear Cell Renal Cell Carcinoma in Mice. Nat. Commun. 11 (1), 4111. doi:10.1038/s41467-020-17873-3

Jahanban-Esfahlan, R., de la Guardia, M., Ahmadi, D., and Yousefi, B. (2018). Modulating Tumor Hypoxia by Nanomedicine for Effective Cancer Therapy. J. Cel Physiol 233 (3), 2019-2031. doi:10.1002/jcp.25859

Jiang, Q., Li, F., Cheng, Z., Kong, Y., and Chen, C. (2020). The Role of E3 Ubiquitin Ligase HECTD3 in Cancer and Beyond. Cell. Mol. Life Sci. 77 (8), 1483-1495. doi:10.1007/s00018-019-03339-3

Knight, M., and Stanley, S. (2019). HIF-1 $\alpha$ as a Central Mediator of Cellular Resistance to Intracellular Pathogens. Curr. Opin. Immunol. 60, 111-116. doi:10.1016/j.coi.2019.05.005

Lee, S.-H., Kang, J. H., Ha, J.-S., Oh, S.-J., Choi, H.-J., Song, J., et al. (2020). Transglutaminase 2-Mediated P53 Depletion Promotes Angiogenesis by Increasing HIF-1 $\alpha$-P300 Binding in Renal Cell Carcinoma. Int. J. Mol. Sci. 21 (14), 5042. doi:10.3390/ijms21145042

Li, Z., Shi, L., Li, X., Wang, X., Wang, H., and Liu, Y. (2021). RNF144A-AS1, a TGF$\beta 1-$ and Hypoxia-Inducible Gene that Promotes Tumor Metastasis and Proliferation via Targeting the miR-30c-2-3p/LOX axis in Gastric Cancer. Cell Biosci 11 (1), 177. doi:10.1186/s13578-021-00689-z
Lin, Y.-J., Shyu, W.-C., Chang, C.-W., Wang, C.-C., Wu, C.-P., Lee, H.-T., et al. (2017). Tumor Hypoxia Regulates Forkhead Box C1 to Promote Lung Cancer Progression. Theranostics 7 (5), 1177-1191. doi:10.7150/thno.17895

Liu, Z., Wang, R., and Zhu, G. (2021). Circ_0035483 Functions as a Tumor Promoter in Renal Cell Carcinoma via the miR-31-5p-Mediated HMGA1 Upregulation. Cancer Manag. Res. 13, 693-706. doi:10.2147/CMAR.S282806

Luo, X., Li, A., Chi, X., Lin, Y., Liu, X., Zhang, L., et al. (2021). Hypoxia-Activated Prodrug Enabling Synchronous Chemotherapy and HIF-1a Downregulation for Tumor Treatment. Bioconjug. Chem. 32 (5), 983-990. doi:10.1021/ acs.bioconjchem.1c00131

Meng, L., Xing, Z., Guo, Z., Qiu, Y., and Liu, Z. (2021). Hypoxia-Induced microRNA-155 Overexpression in Extracellular Vesicles Promotes Renal Cell Carcinoma Progression by Targeting FOXO3. Aging 13, 9613-9626. doi:10.18632/aging.202706

Qin, Z., Hu, H., Sun, W., Chen, L., Jin, S., Xu, Q., et al. (2021). miR-224-5p Contained in Urinary Extracellular Vesicles Regulates PD-L1 Expression by Inhibiting Cyclin D1 in Renal Cell Carcinoma Cells. Cancers 13 (4), 618. doi:10.3390/cancers 13040618

Rankin, E. B., Nam, J.-M., and Giaccia, A. J. (2016). Hypoxia: Signaling the Metastatic Cascade. Trends Cancer 2 (6), 295-304. doi:10.1016/ j.trecan.2016.05.006

Rustum, Y., Chintala, S., Durrani, F., and Bhattacharya, A. (2018). Non-Coding Micro RNAs and Hypoxia-Inducible Factors Are Selenium Targets for Development of a Mechanism-Based Combination Strategy in Clear-Cell Renal Cell Carcinoma-Bench-To-Bedside Therapy. Int. J. Mol. Sci. 19 (11), 3378. doi:10.3390/ijms19113378

Saliminejad, K., Khorram Khorshid, H. R., Soleymani Fard, S., and Ghaffari, S. H. (2019). An Overview of microRNAs: Biology, Functions, Therapeutics, and Analysis Methods. J. Cel Physiol 234 (5), 5451-5465. doi: $10.1002 /$ jcp. 27486

Schödel, J., Grampp, S., Maher, E. R., Moch, H., Ratcliffe, P. J., Russo, P., et al. (2016). Hypoxia, Hypoxia-Inducible Transcription Factors, and Renal Cancer. Eur. Urol. 69 (4), 646-657. doi:10.1016/j.eururo.2015.08.007

Serocki, M., Bartoszewska, S., Janaszak-Jasiecka, A., Ochocka, R. J., Collawn, J. F., and Bartoszewski, R. (2018). miRNAs Regulate the HIF Switch during Hypoxia: A Novel Therapeutic Target. Angiogenesis 21 (2), 183-202. doi:10.1007/s10456018-9600-2

Shingarev, R., and Jaimes, E. A. (2017). Renal Cell Carcinoma: New Insights and Challenges for a Clinician Scientist. Am. J. Physiology-Renal Physiol. 313 (2), F145-F154. doi:10.1152/ajprenal.00480.2016

Song, E., Song, W., Ren, M., Xing, L., Ni, W., Li, Y., et al. (2018). Identification of Potential Crucial Genes Associated with Carcinogenesis of clear Cell Renal Cell Carcinoma. J. Cel. Biochem. 119 (7), 5163-5174. doi:10.1002/jcb.26543

Su, Z. H., Liao, H. H., Lu, K. E., Chi, Z., Qiu, Z. Q., Jiang, J. M., et al. (2020). Hypoxia-Responsive miR-346 Promotes Proliferation, Migration, and Invasion of Renal Cell Carcinoma Cells via Targeting NDRG2. Neoplasma 67 (5), 1002-1011. doi:10.4149/neo_2020_190917N915

Sun, T., Wang, X., He, H. H., Sweeney, C. J., Liu, S. X., Brown, M., et al. (2014). MiR-221 Promotes the Development of Androgen Independence in Prostate Cancer Cells via Downregulation of HECTD2 and RAB1A. Oncogene 33 (21), 2790-2800. doi:10.1038/onc.2013.230

Suo, C., Deng, W., Vu, T. N., Li, M., Shi, L., and Pawitan, Y. (2018). Accumulation of Potential Driver Genes with Genomic Alterations Predicts Survival of HighRisk Neuroblastoma Patients. Biol. Direct 13 (1), 14. doi:10.1186/s13062-0180218-5

Wan, S. S., Yun, B. D., Song, M. G., Lee, J. K., Choi, S. Y., Kuh, H. J., et al. (2021). The Hypoxia-Long Noncoding RNA Interaction in Solid Cancers. Int. J. Mol. Sci. 22 (14), 7261. doi:10.3390/ijms22147261

Wang, X., De Geyter, C., Jia, Z., Peng, Y., and Zhang, H. (2020). HECTD1 Regulates the Expression of SNAIL: Implications for EpithelialMesenchymal Transition. Int. J. Oncol. 56 (5), 1186-1198. doi:10.3892/ ijo. 2020.5002

White, N. M., and Yousef, G. M. (2010). MicroRNAs: Exploring a New Dimension in the Pathogenesis of Kidney Cancer. BMC Med. 8, 65. doi:10.1186/1741-70158-65

Wolf, M. M., Kimryn Rathmell, W., and Beckermann, K. E. (2020). Modeling Clear Cell Renal Cell Carcinoma and Therapeutic Implications. Oncogene 39 (17), 3413-3426. doi:10.1038/s41388-020-1234-3 
Wu, D., Li, M., Wang, L., Zhou, Y., Zhou, J., Pan, H., et al. (2014). microRNA-145 Inhibits Cell Proliferation, Migration and Invasion by Targeting Matrix Metallopeptidase-11 in Renal Cell Carcinoma. Mol. Med. Rep. 10 (1), 393-398. doi:10.3892/mmr.2014.2149

Wu, G., Ke, J., Wang, J., Zhu, X., and Xiong, S. (2020). LncRNA CCAT2 Promotes the Proliferation and Invasion of Renal Cell Cancer by Sponging miR-320a. Panminerva Med. Advance online publication. doi:10.23736/S00310808.19.03778-9

Xiang, M., Huang, Y., Dai, C., and Zou, G. (2021). MiR-340 Regulates the Growth and Metabolism of Renal Cell Carcinoma Cells by Targeting Frizzled Class Receptor 3. Arch. Pharm. Res. 44 (2), 219-229. doi:10.1007/s12272-02101310-0

Xing, B., Qiao, X.-F., Qiu, Y.-H., and Li, X. (2021). TMPO-AS1 Regulates the Aggressiveness-Associated Traits of Nasopharyngeal Carcinoma Cells through Sponging miR-320a. Cancer Manag. Res. 13, 415-425. doi:10.2147/ CMAR.S285113

Yang, S.-L., Liu, L.-P., Jiang, J.-X., Xiong, Z.-F., He, Q.-J., and Wu, C. (2014). The Correlation of Expression Levels of HIF-1 and HIF-2 in Hepatocellular Carcinoma with Capsular Invasion, Portal Vein Tumor Thrombi and Patients' Clinical Outcome. Jpn. J. Clin. Oncol. 44 (2), 159-167. doi:10.1093/ jjco/hyt194

Yang, Y., Qu, A., Wu, Q., Zhang, X., Wang, L., Li, C., et al. (2020). Prognostic Value of a Hypoxia-Related microRNA Signature in Patients with Colorectal Cancer. Aging 12 (1), 35-52. doi:10.18632/aging.102228

Yang, Y., Liu, L., Sun, J., Wang, S., Yang, Z., Li, H., et al. (2021). Deoxypodophyllotoxin Inhibits Non-Small Cell Lung Cancer Cell Growth by Reducing HIF-1a-Mediated Glycolysis. Front. Oncol. 11, 629543. doi:10.3389/fonc.2021.629543

Yin, J., Ge, X., Shi, Z., Yu, C., Lu, C., Wei, Y., et al. (2021). Extracellular Vesicles Derived from Hypoxic Glioma Stem-Like Cells Confer Temozolomide Resistance on Glioblastoma by Delivering miR-30b-3p. Theranostics 11 (4), 1763-1779. doi:10.7150/thno.47057

Zhang, W., Yang, H., Wang, Z., Wu, Y., Wang, J., Duan, G., et al. (2021). miR-320a/ SP1 Negative Reciprocal Interaction Contributes to Cell Growth and Invasion in Colorectal Cancer. Cancer Cel Int 21 (1), 175. doi:10.1186/s12935-02101874-3

Zhang, L., Chen, H., He, F., Zhang, S., Li, A., Zhang, A., et al. (2021). MicroRNA-320a Promotes Epithelial Ovarian Cancer Cell Proliferation and Invasion by Targeting RASSF8. Front. Oncol. 11, 581932. doi:10.3389/ fonc. 2021.581932

Zhang, C., Pan, X., Peng, X., Liu, K., Wang, J., Zhao, L., et al. (2021). miR-30b-5p Up-Regulation Related to the Dismal Prognosis for Patients with Renal Cell Cancer. J. Clin. Lab. Anal. 35 (2), e23599. doi:10.1002/jcla.23599

Zhao, S., Wang, Y., Lou, Y., Wang, Y., Sun, J., Luo, M., et al. (2018). MicroRNA320a Suppresses Tumour Cell Proliferation and Invasion of Renal Cancer Cells by Targeting FoxM1. Oncol. Rep. 40 (4), 1917-1926. doi:10.3892/or.2018.6597

Zhou, W., Zhong, Z., Lin, D., Liu, Z., Zhang, Q., Xia, H., et al. (2021). Hypothermic Oxygenated Perfusion Inhibits HECTD3-Mediated TRAF3 Polyubiquitination to Alleviate DCD Liver Ischemia-Reperfusion Injury. Cel Death Dis 12 (2), 211. doi:10.1038/s41419-021-03493-2

Conflict of Interest: The authors declare that the research was conducted in the absence of any commercial or financial relationships that could be construed as a potential conflict of interest.

Publisher's Note: All claims expressed in this article are solely those of the authors and do not necessarily represent those of their affiliated organizations, or those of the publisher, the editors and the reviewers. Any product that may be evaluated in this article, or claim that may be made by its manufacturer, is not guaranteed or endorsed by the publisher.

Copyright (C) 2021 Lv, Shen, Yao, Yang, Xiang and Ma. This is an open-access article distributed under the terms of the Creative Commons Attribution License (CC BY). The use, distribution or reproduction in other forums is permitted, provided the original author(s) and the copyright owner(s) are credited and that the original publication in this journal is cited, in accordance with accepted academic practice. No use, distribution or reproduction is permitted which does not comply with these terms. 